\title{
Charge-Transfer Intermediates in the Electrochemical Doping Mechanism of Conjugated Polymers
}

\author{
Ilaria Bargigia, ${ }^{\dagger, \AA}$ Lisa R. Savagian, ${ }^{\ddagger}, \mathbb{I}$ Anna M. Österholm, ${ }^{\dagger}$ \\ John R. Reynolds, ${ }^{\dagger, \dagger}$ and Carlos Silva ${ }^{*, \dagger, \S, \ddagger}$ \\ $\dagger$ School of Chemistry and Biochemistry, Georgia Tech Polymer Network, Center for Organic \\ Photonics and Electronics, Georgia Institute of Technology, 901 Atlantic Drive, \\ Atlanta, GA 30332, United States \\ $\$$ School of Material Science and Engineering, Georgia Institute of Technology, \\ 901 Atlantic Drive, Atlanta, GA 30332, United States \\ $\S$ School of Physics, Georgia Institute of Technology, Center for Organic Photonics \\ and Electronics, 837 State Street NW, Atlanta, \\ GA 30332, United States \\ IThese authors contributed equally to this work. \\ E-mail: carlos.silva@gatech.edu
}

\begin{abstract}
In this work, we address the nature of electrochemically induced charged states in conjugated polymers, their evolution as a function of electrochemical potential, and their coupling to their local environment by means of transient absorption and Raman spectroscopies synergistically performed in situ throughout the electrochemical doping process. In particular, we investigate the fundamental mechanism of electrochemical doping in an oligoether-functionalized 3,4propylenedioxythiophene (ProDOT) copolymer. The changes embedded in both linear and transient absorption features allow us to identify a precursor electronic state with charge-transfer (CT) character that precedes polaron formation and bulk electronic conductivity. This state is shown to contribute to the ultrafast quenching of both neutral molecular excitations and polarons.
\end{abstract}


Raman spectra relate the electronic transition of this precursor state predominantly to the $\mathrm{C}_{\beta}-\mathrm{C}_{\beta}$ stretching mode of the thiophene heterocycle. We characterize the coupling of the CT-like state with primary excitons and electrochemically induced charge-separated states, providing insight into the energetic landscape of a heterogeneous polymer-electrolyte system and demonstrate how such coupling depends on environmental parameters, such as polymer structure, electrolyte composition, and environmental polarity.

\section{Introduction}

Organic mixed ionic-electronic conductors (OMIECs) constitute a broad family of mostly $\pi$ conjugated polymers with the remarkable ability to support facile and simultaneous transport of ionic and electronic charged species. During electrochemical doping, charge carriers are formed along the conjugated polymer backbone in response to a sufficiently large change in potential of the underlying electrode to initiate a charge transfer across the OMIEC-electrode interface. Simultaneously, solvated electrolyte counter ions move across the OMIEC-electrolyte interface and into the bulk of the active OMIEC layer to stabilize the induced charge carriers. Accordingly, the transport and coupling of ionic and electronic species in OMIECs involves interactions and processes spanning multiple length scales and interfaces. As such, mixed transport in conjugated polymers is influenced by an assortment of factors, including the choice of polymer structure, the extent of inter- and intrachain electronic coupling, the mesoscale assembly of polymer chains in the active layer, the ability of the polymer to interact with its ionic environment, and the nature of the polymer-electrode interface.

Future technological development relies on understanding both ion and electron transport individually along with the coupling of these two properties and the elementary processes that govern charge formation and stabilization. ${ }^{1-3}$ Such information is relevant across a wide range of applications that rely on OMIECs, such as electrochemical charge storage devices (batteries and supercapacitors), ${ }^{4-8}$ electrochromic displays, ${ }^{9-11}$ light-emitting electrochemical cells, ${ }^{12,13}$ as well as several emerging bioelectronic technologies, ${ }^{3,14}$ including organic electrochemical transistors (OECTs), ${ }^{15-19}$ ion pumps, ${ }^{20,21}$ sensors, ${ }^{22,23}$ and soft actuators. ${ }^{24,25}$ Regardless of the application, all of these devices involve polymeric active layers that undergo continuous changes in their oxidation state (doping level) due to local variations in electrochemical potential. Such doping processes 
result in significant, potential-dependent fluctuations in polymer microstructure, energetic landscape, electrolyte solvation, ion content, and electronic density of states. An additional layer of complexity is introduced by the weak intermolecular coupling between polymer chains and numerous degrees of conformational freedom in organic materials. Combined with the high charge density required for electrochemical doping, this often yields heterogeneous potential energy landscapes in the OMIEC, where different regions or domains of a single active layer can vary greatly in terms of their electroactivity and charge transport properties. In some cases, heterogeneity occurs over extremely small length scales comprising only a few chromophore segments, which greatly limits the utility of conventional characterization techniques that rely on longer-range order and/or periodicity (e.g. X-ray and scanning probe methods ${ }^{26-30}$ ) for understanding the doping process. It is therefore necessary to develop new approaches for capturing the dynamic picture underlying electrochemical doping to provide a molecular description of charged states in weakly-coupled, structurally irregular, and heterogeneous polymer-electrolyte systems. Specifically, a molecular-level understanding of the nature of all electronic species involved in the electrochemical doping process, including electronic intermediates and their relationship to fully de-doped and fully doped states, is required for the rational design of novel OMIECs with the greatest performance in wide-ranging applications.

To address part of this growing challenge, we apply a unique combination of both linear and ultrafast spectroscopies in situ to probe the nature and dynamics of the primary photoexcitations and the ground-state polaron population in a model OMIEC while modulating its electrochemical doping level. Investigating the excited-state dynamics of photoexcitations in this manner allows us to gain insight into the formation and evolution of charge carriers, the coupling of such excitations to their environment (i.e. molecular vibrations, electronic fluctuations, electrolyte composition, other excited states, etc.), and the interaction of these states with neighboring excitons and polarons - all as a function of the electrochemical potential.

Here, we characterize the dynamics of primary photoexcitations in an oligoetherfunctionalized 3,4-propylenedioxythiophene copolymer (ProDOT(OE 3 -DMP) and evaluate the correlation of these excitations with the evolution of macromolecular and environmental properties as the doping process unfolds. As previously reported, ProDOT(OE 3$)-D M P$ can be fully and reversibly p-doped in both aqueous and organic electrolytes due to its electronic-rich and sterically 
protected backbone, low oxidation potential, and polar side chains, ${ }^{31}$ making this material uniquely suited for these studies. Incorporation of oligoether groups, which facilitate electrochemical doping in aqueous media, is a common structural approach for designing high performance OMIECs, especially in devices that require electrochemical interfacing with biological systems. ${ }^{32-}$ ${ }^{36}$ By performing measurements in situ with precise control of the polymer's doping level, we develop an understanding regarding the elementary formation of charge carriers and their coupling to their local environment in a model p-type OMIEC.

In particular, we present results demonstrating that the electrochemical doping of ProDOT $\left(\mathrm{OE}_{3}\right)$-DMP is mediated by a sub-gap state which emerges prior to the formation of mobile charge carriers. This state exhibits properties consistent with a polarized molecular excitation, i.e. a fundamental electronic excitation that has acquired a charge-transfer (CT) character, in which the electron and hole density is weighted unequally in different parts of the chromophore. Assignment of this species is supported by resonance Raman, linear absorption, and transient absorption spectroscopies performed incrementally throughout the electrochemical doping process. Together, these techniques show that this CT-like state $(i)$ is present prior to the onset of redox current and is highly sensitive to the chemical composition of the surrounding environment, (ii) exhibits a potential dependence distinct from the traditionally assigned "polaron" feature, ${ }^{37}$ (iii) shares a common ground state with the exciton of the undoped polymer, and (iv) plays a substantial role in the ultrafast quenching of the excitonic population. Depending on the environmental parameters (protic and aprotic systems, ion size and structure, polarity, hydrogen bonding, $\mathrm{pH}$, etc.), this state could play an active role in the doping mechanism by favoring the reorganization of the electronic configuration from the neutral to the doped form: understanding its nature, coupling to the environment, and relationship to other electronic species is therefore a necessary step for an informed design of novel architectures for specific applications. ${ }^{1}$

Our proposed mechanism of the electrochemical doping of ProDOT(OE 3$)-\mathrm{DMP}$, as presented in Figure 1, will be elaborated through the discussion of the experimental results and the implications thereof. We highlight the significance of these interpretations as they relate to the optoelectronic properties of electrochemically induced charges and their interaction with neutral excitons, especially in the context of recent literature concerning the excited state dynamics of an 
acid-doped polyelectrolyte. ${ }^{38}$ These foundational studies demonstrate the utility and application of in situ ultrafast and nonlinear spectroscopy in understanding redox processes in OMIECs.

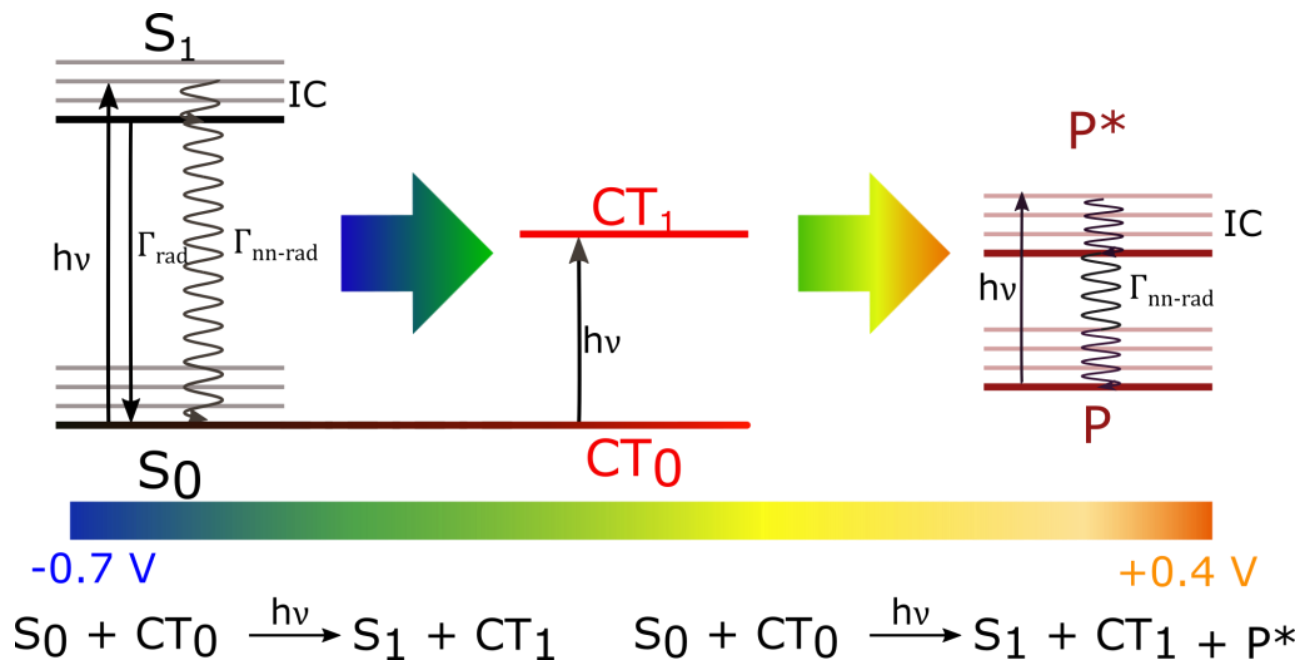

Figure 1: Schematic representation of the electronic states in ProDOT( $\left.\mathrm{OE}_{3}\right)-\mathrm{DMP}$ and their evolution with doping. In the neutral state the primary photoexcitation is constituted by singlet excitons $\left(S_{1}\right)$. A polarized molecular excitation, denoted as a charge transfer state (CT), shares a common ground state with the exciton, appears before substantial doping, and acts as a precursor to polaron $(\mathrm{P})$ formation. Pathways of radiative decay/fluorescence $\left(\Gamma_{\mathrm{rad}}\right)$, nonradiative decay $\left(\Gamma_{\mathrm{nn}}\right.$ rad), and internal conversion (IC) are labeled.

\section{Experimental Section}

\section{Film preparation}

The polymer was synthesized via direct (hetero)arylation polymerization by copolymerizing a 3,4-propylenedioxythiophene (ProDOT) comonomer (difunctionalized at the 2-position of the propylene bridge with two oligoether $\left(\mathrm{OE}_{3}\right)$ chains) with a 3,3-dimethyl-3,4-dihydro-2Hthieno[3,4-b][1,4]dioxepine comonomer (referred to as 2,2-dimethyl-ProDOT or DMP). The resulting polymer is referred to here as $\operatorname{ProDOT}\left(\mathrm{OE}_{3}\right)-\mathrm{DMP}$. The repeat unit structure is shown in Figure 2A. The molecular weight of the polymer was determined to be $\mathrm{M}_{n}=23 \mathrm{kDa}(\mathrm{Ð}=2.0)$ by GPC in $\mathrm{CHCl}_{3}\left(40{ }^{\circ} \mathrm{C}\right)$ vs. a polystyrene standard. The synthetic conditions, ${ }^{1} \mathrm{H}-\mathrm{NMR},{ }^{13} \mathrm{C}-\mathrm{NMR}$, elemental analysis, and size exclusions chromatograms can be found in previous literature. ${ }^{31}$ Due 
to the polar oligoether chains and electron-rich backbone, this polymer supports the transport of hydrated/solvated ions and is highly redox active in not only organic electrolytes but also aqueous electrolytes and buffers. Accordingly, films of this material can be fully electrochemically p-doped (complete depletion of the neutral $\pi-\pi^{*}$ optical transition) within the electrochemical window of water. For all in situ characterizations, thin films of ProDOT(OE 3$)$-DMP were spray-cast from room-temperature chloroform solutions $\left(4 \mathrm{mg} \mathrm{mL}^{-1}\right)$ onto ITO/glass slides $\left(8-12 \mathrm{ohm} \mathrm{sq}^{-1}\right)$, Delta Technologies) using an Iwata-Eclipse HP-BC airbrush with argon carrier gas (20 psi). Films with thicknesses ranging from 100-200 nm were obtained. Prior to coating, ITO/glass substrates were cleaned by sonication in sodium dodecyl sulfate in deionized water, deionized water, acetone, and isopropanol, then dried under an argon stream.

\section{X-Ray Scattering}

Films of ProDOT $\left(\mathrm{OE}_{3}\right)$-DMP were spray-cast as previously described onto polished silicon wafers. Prior to coating samples, the wafers were cleaned with Piranha etch, then rinsed with distilled water and dried under an argon stream. Grazing incidence wide angle X-ray scattering (GIWAXS) was performed at the Stanford Synchrotron Radiation Lightsource on Beamline 11-3. The sample was irradiated with an incident beam energy of $12.7 \mathrm{keV}$ with a sample distance of $250 \mathrm{~mm}$. Calibration of the scattering profile was performed using a standard sample of $\mathrm{LaB}_{6}$. More information about GIWAXS data analysis can be found in the Supporting Information (SI Figure S1, SI Table S1).

\section{UV-Vis-NIR Spectroelectrochemistry}

Polymer-coated ITO/glass slides were immersed in a standard three-electrode electrochemical cell containing $0.5 \mathrm{M} \mathrm{NaCl}$ (biotechnology grade, Amresco) dissolved in ultrapure $\mathrm{H}_{2} \mathrm{O}(>18.2$ $\mathrm{M} \Omega)$, a platinum flag counter electrode, and a reference electrode ( $\mathrm{Ag} / \mathrm{AgCl}, 3 \mathrm{M}$ aqueous $\mathrm{NaCl}$ inner solution, BASi, Inc.). Electrolyte $\mathrm{pH}$ conditions evaluated using Micro Essential Lab Hydrion Insta-Check pH Test Paper (Ficher Sci.). Before recording UV-Vis-NIR absorbance spectra in the aqueous media, the films were electrochemically conditioned by cycling them between -0.8 and $+0.8 \mathrm{~V}$ vs. $\mathrm{Ag} / \mathrm{AgCl}$ for 10 cycles at $50 \mathrm{mV} \mathrm{s}^{-1}$. Potential-dependent linear absorption spectra were recorded using a Cary 5000 UV-Vis-NIR spectrophotometer (300-1400 $\mathrm{nm}, 2000 \mathrm{~nm} \mathrm{~s}^{-1}$ ) using a quartz cuvette (path length of $1 \mathrm{~cm}$ ) under ambient conditions. The 
electrochemical cell was addressed using an EG\&G Princeton Applied Research model 273A potentiostat/galvanostat under CorrWare control. For experiments performed in an organic electrolyte, films were prepared in the same manner and incorporated into a three-electrode cell containing a $0.5 \mathrm{M}$ tetrabutylammonium hexafluorophophate (TBAPF6, Acros Organics, 98\%, purified by recrystallization from hot ethanol) solution in propylene carbonate (PC, Acros organics, $99.5 \%$, purified using a solvent purification system). The counter electrode was a platinum flag, and the reference electrode was a $\mathrm{Ag} / \mathrm{Ag}^{+}$pseudoreference electrode $(10 \mathrm{mM}$ $\mathrm{AgNO}_{3}$ in $0.5 \mathrm{M} \mathrm{TBAPF}_{6}$ in acetonitrile as the inner solution, $E_{1 / 2}$ for $\mathrm{Fc} / \mathrm{Fc}^{+}$couple $=+0.056 \mathrm{~V}$ in a $0.5 \mathrm{M} \mathrm{TBAPF}_{6} / \mathrm{PC}$ electrolyte solution under ambient conditions. Prior to spectroelectrochemical measurements, films were electrochemically conditioned by cycling them between -0.80 and +0.80 $\mathrm{V} v$ s. the $\mathrm{Ag} / \mathrm{Ag}^{+}$reference for 10 cycles at $50 \mathrm{mV} \mathrm{s}^{-1}$. Prior to recording all spectra, films were held at the indicated electrochemical conditions for 15-30 seconds until the current transient reached a steady state, indicating an equilibrated oxidation level. Cyclic voltammograms are shown for films spray-cast on ITO/glass in the indicated electrolyte, recorded at $50 \mathrm{mV} \mathrm{s}^{-1}$. The reported voltammograms represent the trace obtained after 5-15 cycles to ensure proper conditioning of the film and reproducible current-voltage characteristics.

\section{Transient Absorption Measurements}

Measurements were performed similarly to the linear spectroelectrochemical measurements described above, using the same cuvette, electrolytes, electrode architecture, and film deposition conditions, as shown in Figure 5A. Prior to data acquisition, freshly cast films were conditioned by repeated electrochemical cycling as described previously. The electrochemical cell was controlled by a Pine WaveNow Potentiostat and AfterMath software. The transient absorption spectroscopy configuration was commercially acquired (Hera system, Light Conversion) and used a $20 \mathrm{~W}$ laser source emitting $\sim 220$ fs pulses at $1030 \mathrm{~nm}$ with $100 \mathrm{kHz}$ repetition rate (Pharos Model PH1-20-0200-02-10, Light Conversion). An optical parametric amplifier (Orpheus, Light Conversion) was used to generate the pump excitation $(2.29 \mathrm{eV}, 542 \mathrm{~nm})$. A single-filament whitelight continuum (1.18-2.53 eV, 490-1050 nm) was generated by focusing $2 \mathrm{~W}$ of the laser light onto a sapphire crystal and used as the probe beam. The probe light transmitted through the sample was dispersed by an imaging spectrograph (Shamrock 193i, Andor Technology Ltd., U.K.) and detected by a multichannel detector. For the transient spectra recorded as a function of the applied 
potential, fast scans of the time delay between pump and probe beams were used, where only three delays were acquired. For the dynamic measurements, the time delay ranged from -1 to +10 ps. The pump excitation densities were scaled according to the loss of absorption measured by linear absorption. For the neutral state of the film, the typical fluence used was $0.2 \mu \mathrm{J} / \mathrm{cm}^{2}$, with a pump beam radius of $\sim 469 \mu \mathrm{m}$ estimated at the $1 / \mathrm{e}^{2}$ plane.

\section{Raman Spectroscopy}

Resonance Raman spectroscopy was conducted at the Center for Nanophase Materials Sciences at Oak Ridge National Laboratory (Oak Ridge, TN). Measurements were performed in situ using a custom-made cell filled with $0.5 \mathrm{M} \mathrm{NaCl} / \mathrm{H}_{2} \mathrm{O}$ electrolyte, as shown in Figure 4A. For sample preparation, films of ProDOT( $\left(\mathrm{OE}_{3}\right)$-DMP were spray-cast on ITO/glass working electrodes as described above. The cell was equipped with the same Pt counter electrode and $\mathrm{Ag} / \mathrm{AgCl}$ reference electrodes identified previously. The polymer-coated working electrode was placed on top of the cell with the polymer-coated side facing downward to contact the electrolyte. The electrochemical cell was controlled by a Pine WaveNow Potentiostat and AfterMath software. The films were electrochemically conditioned in $0.5 \mathrm{M} \mathrm{NaCl} / \mathrm{H}_{2} \mathrm{O}$ as previously described and allowed to equilibrate for 10-30 seconds at each potential prior to spectral acquisition.

Raman spectra were collected in reflectance mode using inVia Renishaw confocal microRaman spectroscopy systems equipped with a $532 \mathrm{~nm}(2.44 \mathrm{eV})$ laser and a $758 \mathrm{~nm}(1.58 \mathrm{eV})$ laser. Raman spectra were collected while applying the indicated electrochemical potential. For both excitation energies, the excitation lasers were focused through the back of the ITO/glass substrate and onto the underlying polymer film using a 20x objective lens (10\% power, exposure time of $10 \mathrm{~s}$, slit opening of $100 \mu \mathrm{m})$. For the $1.58 \mathrm{eV}$ excitation, spectra were collected from 100$3000 \mathrm{~cm}^{-1}$ using a grating with 1200 lines $\mathrm{mm}^{-1}$. For the $2.44 \mathrm{eV}$ excitation, spectra were collected from 8-2000 $\mathrm{cm}^{-1}$ using a grating with 3000 lines $\mathrm{mm}^{-1}$. For each measurement, 6-9 individual acquisitions were performed on different points on the film and averaged. The potential-dependent spectra for multiple films were collected to ensure reproducibility. Representative Raman spectra for each excitation wavelength correspond to data taken with two separate films of comparable thicknesses. Data analyses (baseline subtraction, cosmic ray removal, and peak fitting) were performed in the Renishaw WiRE 5 software. 


\section{Results and Discussion}

\section{Microstructural Analysis}

The factors governing charge carrier generation, stabilization, and transport in conjugated polymers are complex, often depending on a range of interrelated chemical, electronic, and structural properties. For neat polymer films in the solid state, the macromolecular arrangement of chain segments is known to play a critical role in shaping the optoelectronic properties of the bulk material. To probe the extent of solid-state ordering in the polar conjugated polymer ProDOT( $\left(\mathrm{OE}_{3}\right)$-DMP (Figure 2A), grazing-incidence wide angle X-ray scattering (GIWAXS) was performed on neat films of the polymer spray-cast from a dilute chloroform solution. The twodimensional GIWAXS diffraction pattern of ProDOT(OE $\left.\mathrm{OE}_{3}\right)$-DMP (Figure 2B) does not suggest the presence of any significant crystalline microstructure or long-range order. The diffracting regions of the film exhibits a slight face-on texturing relative to the substrate, as indicated by the notable out-of-plane $\left(q_{z}\right)$ scattering at $q_{z}=1.57 \AA^{-1}$, which is attributed to scattering from $\pi$ stacks (labeled (020) in Figure 2B, $d_{020}=3.99 \AA$ ). This feature is superimposed on a broad, amorphous scattering halo, commonly attributed to side chain disorder in alkyl-functionalized polythiophenes. ${ }^{39}$ Scattering in the $q_{z}$ direction at $\sim 0.296 \AA^{-1}$ is attributed to the lamellar stacking (denoted (100) in Figure 2B) and corresponds to a distance between adjacent polymer chains of $d_{100}=21.2 \AA$.

The extent of disorder in any given crystallographic direction can be quantified by the paracrystallinty parameter $g$, which encompasses static fluctuations around a given lattice spacing. Here, we find the $g$ for ProDOT( $\left(\mathrm{OE}_{3}\right)$-DMP to be approximately $19 \%$ in the $(0 \mathrm{kO})$ direction (SI Table S1), which indicates an amorphous film microstructure ${ }^{40}$ and suggests that the intermolecular order associated with $\pi$-stacks is not maintained for an appreciable length scale. For comparison, semicrystalline poly(3-hexylthiophene) (P3HT) typically exhibits relatively tighter $\pi$-stacking distances $\left(d_{020}=\text { c. } 3.8 \AA\right)^{28,39,41-43}$ as well as much lower paracrystallinity in the $(\mathrm{OkO})$ direction ( $g=4-7 \%,{ }^{44}$ depending on molecular weight and processing conditions), indicating significantly less disorder in the system. 
The 2D GIWAXS pattern of spray-cast ProDOT(OE) short-range, weakly diffracting aggregates. Aggregates, which are distinct from crystallites, are comprised of chain segments that exhibit short-range order in only one dimension (e.g. $\pi$-stacking) without participating in complementary structure along crystallographic directions (e.g. lamellar ordering) ${ }^{45}$ For ProDOT( $\left(\mathrm{OE}_{3}\right)$-DMP, such aggregation is apparent from the scattering pattern (Figure 2B) and corrected line cuts (SI Figure S1), which show weak out-of-plane $\pi-\pi$ scattering features that are not complemented by any in-plane lamellar structures of similar scattering intensity. While aggregates are necessary for the formation of bulk crystallites, they may also exist in amorphous matrices. The GIWAXS pattern for ProDOT( $\left(\mathrm{OE}_{3}\right)$-DMP suggests that, unlike many widely-studied conjugated polymers, this material does not assemble into domains with intermolecular order spanning multiple dimensions. Rather, we see that any ordering of chain segments in ProDOT $\left(\mathrm{OE}_{3}\right)$-DMP is incredibly short range and confined to one dimension only. The lack of multi-dimensional ordering observed in ProDOT( $\left(\mathrm{OE}_{3}\right)$-DMP is confirmed by the absence of any prominent thermal transitions in differential scanning calorimetry (SI Figure S2), as reported previously. ${ }^{31}$ Accordingly, X-ray methods are not expected to be particularly powerful tools for developing a structural picture of charged states in this material. We therefore employ in situ spectroscopic techniques as more relevant, molecular-level methods for understanding the electrochemical doping of ProDOT( $\left.\mathrm{OE}_{3}\right)$-DMP. 
A. $\operatorname{ProDOT}\left(\mathrm{OE}_{3}\right)$-DMP

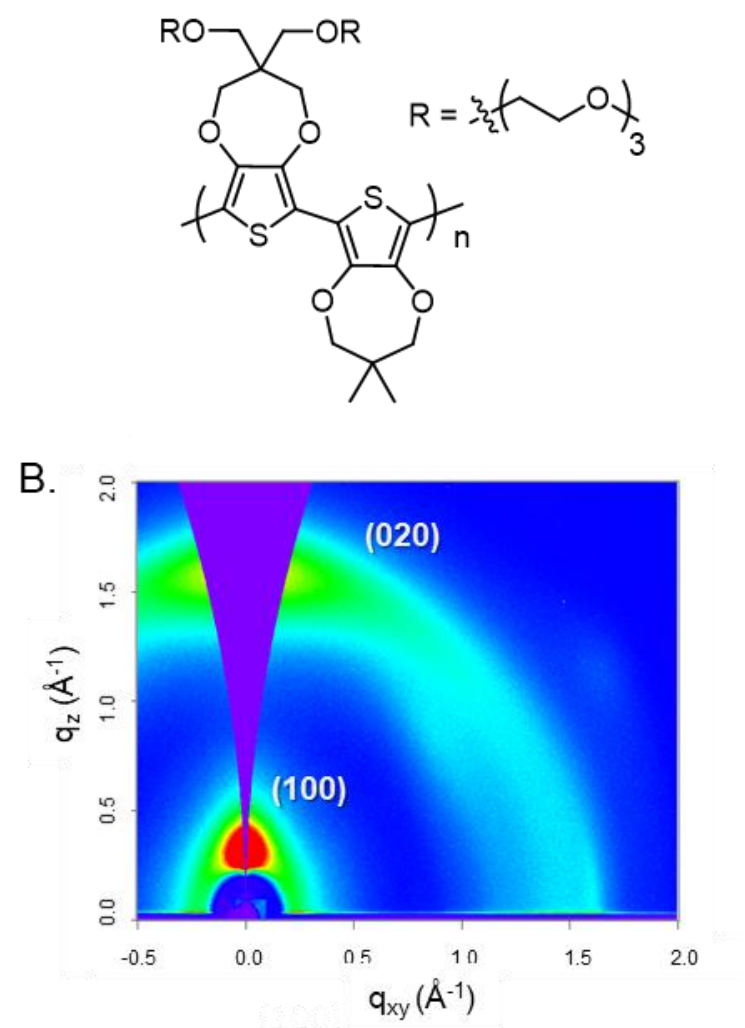

Figure 2: (A) Repeat unit structure of ProDOT( $\left.\mathrm{OE}_{3}\right)$-DMP. (B) Two-dimensional GIWAXS diffraction patterns of spray-cast films of $\operatorname{ProDOT}\left(\mathrm{OE}_{3}\right)$-DMP on polished silicon, with lamellar (100) and $\pi-\pi(020)$ diffraction arcs labeled. Scattering intensity is represented linearly on a blue (low) to red (high) color scale.

\section{Electrochemical Doping of ProDOT(OE 3$)-D M P$}

Next, we focus on the redox behavior of $\operatorname{ProDOT}\left(\mathrm{OE}_{3}\right)$-DMP and its potential-dependent linear absorption spectra to understand the doping processes in this material. Electrochemical doping affords a precise and highly controlled means to modify the charge-carrier density (and therefore the oxidation state or doping level) of conjugated polymers. Figure 3A shows the cyclic voltammogram (CV) of a spray-cast ProDOT( $\left(\mathrm{OE}_{3}\right)$-DMP film in ambient $0.5 \mathrm{M} \mathrm{NaCl} / \mathrm{H}_{2} \mathrm{O}$. The potential sweep ranges from $-0.8 \mathrm{~V}$ vs. $\mathrm{Ag} / \mathrm{AgCl}$, where the film is in its charge-neutral state, to 
$+0.8 \mathrm{~V}$, which is sufficiently high to convert the film to its p-doped form while avoiding concomitant water electrolysis. The current response shows two broad redox processes with an onset of oxidation at approximately $-0.21 \mathrm{~V}$. The onset of oxidation depends not only on the ionization energy of the polymer but also on the ability of the polymer to delocalize charge carriers along and across chains, the favorability of electron transfer at the polymer-electrode interface, and the ability of the electrolyte to penetrate the polymer film and allow for facile ion transport in and out of the film. Here, the low oxidation potential of $\operatorname{ProDOT}\left(\mathrm{OE}_{3}\right)$-DMP is due to a combination of $\pi$ electron-donating oxygen atoms in the $\beta$-positions of the thiophene ring that increase the overall electron density of the conjugated backbone, as well as the polar side chains that promote the uptake of water and hydrated ions. ${ }^{31,32}$ Following the principal onset of oxidation, an additional oxidation wave is observed, with an onset of approximately $+0.2 \mathrm{~V}$ and a peak current observed at $+0.45 \mathrm{~V}$. In alkyl-functionalized polythiophenes and similar ProDOT-based copolymers, multiple redox peaks have been proposed to arise from the presence of electronically resolved subpopulations that vary in their interchain packing and disorder that give rise to the coexistence of film domains with different potential energy landscapes. ${ }^{46-48}$

We can utilize the fact that the neutral and charged states of the polymer exhibit characteristic optical absorption bands in the visible and near-infrared (NIR) regions by tracking the potentialdependent optical lineshape with linear spectroscopy. This allows us to monitor the evolution and extent of the electrochemical doping reaction. Linear absorption spectra of a ProDOT(OE 3$)$-DMP film were recorded in $0.1 \mathrm{~V}$ increments from $-0.7 \mathrm{~V}$ to $+0.8 \mathrm{~V}$ (Figure 3B, plotted in logarithmic scale; see SI Figure S3 for spectra plotted on a linear scale). The absorbance signature of the neutral polymer exciton appears in the $1.8-3.0 \mathrm{eV}$ energy region, showing vibronic features $A_{0-0}$ at $\sim 1.94$ $\mathrm{eV}, \mathrm{A}_{0-1}$ at $\sim 2.13 \mathrm{eV}$, and a high energy shoulder $A_{0-2}$ at $\sim 2.32 \mathrm{eV}$. During the initial portion of the potential sweep from -0.7 to $-0.2 \mathrm{~V}$ (Figure 3A, Region I, highlighted in blue), the linear spectra show the neutral exciton as well as two small absorbance features in the NIR at $\sim 1.58 \mathrm{eV}$ and $\sim 1.11 \mathrm{eV}$. Immediately prior to the onset of oxidation (ca. $-0.21 \mathrm{~V}$ ), these two spectral features in the NIR gain strength, which is accompanied by a moderate bleaching of the neutral ground state. Such features continue to grow immediately after the onset of the doping process between $-0.20 \mathrm{~V}$ and $0.00 \mathrm{~V}$. In addition, we observe that the feature at $\sim 1.11 \mathrm{eV}$ shows a vibronic structure (SI Figures S3, S8). This electron-vibrational coupling is affected by doping; an empirical baseline 
subtraction shows that the vibronic progression associated with this state blue-shifts, and that the peak at the lowest energy loses relative intensity as the doping proceeds (SI Figure S8).

Given that these features exist already in a potential range where there is negligible redox current, they cannot be representative of polarons that form upon oxidative doping. ${ }^{37}$ Moreover, we observe that the intensity of these transitions in the de-doped polymer depends on subtle changes in the $\mathrm{pH}$ of aqueous electrolyte, with more acidic aqueous media giving rise to increased absorbance at 1.58 and $1.11 \mathrm{eV}$ (SI Figure S4). Meanwhile, the choice of processing method (spray $v s$. blade coating) shows minimal impact on the presence of these sub-gap features (SI Figure S4). To exclude the possibility that these features are associated with any type of protic acid doping process unique to aqueous media, we also evaluated the cyclic voltammetry and potentialdependent spectra for ProDOT(OE 3 -DMP in a polar aprotic electrolyte solution of $\mathrm{TBAPF}_{6}$ in propylene carbonate (PC). In this environment, such features are still evident and grow in intensity up to 0.2 V prior to the onset of oxidation (SI Figure S5). As a structural comparison, we examine the cyclic voltammetry and potential-dependent spectra for two similar ProDOT-based copolymers functionalized with aliphatic and ester side chains that are considerably less polar than the oligoether chains in ProDOT(OE 3$)$-DMP. When doping these less polar polymers in the polar aprotic medium, we still observe sub-gap such features at $\sim 1.6 \mathrm{eV}$. However, these features are slightly blue-shifted, are less intense in the de-doped state, and exhibit a slightly different potential dependence when compared to the sub-gap states observed for ProDOT(OE $\mathrm{O}_{3}$ )-DMP (SI Figures S6-S7). From these observations, we conclude that the energy and intensity of these sub-gap species are highly dependent on the environment and appear to be stabilized by polarity, be it polarity induced by electrolyte composition or polymer structure. We will expand on the description of these NIR features later in the manuscript, focusing on the transition at $1.58 \mathrm{eV}$. 
A.

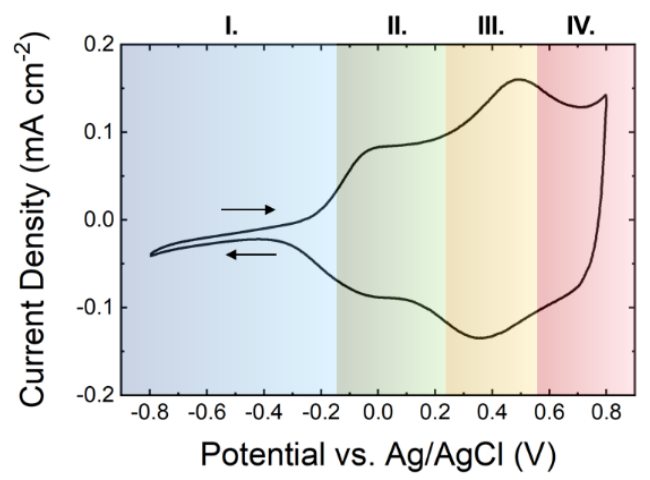

B.

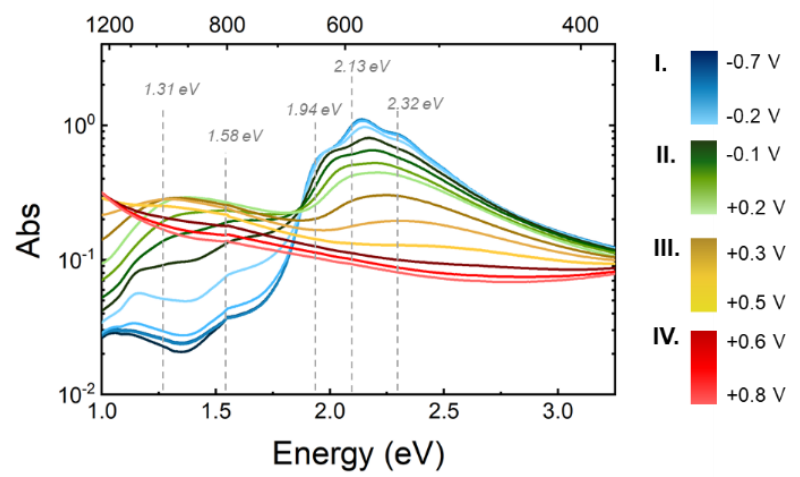

Figure 3: (A) Cyclic voltammogram of ProDOT(OE 3 )-DMP on ITO/glass in $0.5 \mathrm{M} \mathrm{NaCl} / \mathrm{H}_{2} \mathrm{O}$ recorded under ambient conditions at $50 \mathrm{mV} \mathrm{s}^{-1}$ from $-0.8 \mathrm{~V}$ to $+0.7 \mathrm{~V}$ vs. $\mathrm{Ag} / \mathrm{AgCl}$. Arrows indicate the direction of the potential waveform. (B) Linear absorption spectra of ProDOT(OE $)_{3}$ DMP as a function of potential from $-0.7 \mathrm{~V}$ to $+0.8 \mathrm{~V}$ in $0.1 \mathrm{~V}$ increments. Potential regions I, II, II, IV are indicated in blue, green, yellow, and red gradients, respectively.

After the onset of oxidation of ProDOT( $\left(\mathrm{OE}_{3}\right)-\mathrm{DMP}$ in $0.5 \mathrm{M} \mathrm{NaCl} / \mathrm{H}_{2} \mathrm{O}(-0.21 \mathrm{~V})$, a significant amount of charge is extracted from the film, as indicated by a high redox current between -0.1 and $+0.3 \mathrm{~V}$ vs. $\mathrm{Ag} / \mathrm{AgCl}$ (Figure 3, Region II, highlighted in green). In this potential range, a new spectral feature emerges in the NIR centered at $\sim 1.31 \mathrm{eV}$. Throughout this redox process, the new feature at $1.31 \mathrm{eV}$ increases in intensity at the expense of the neutral state absorption through an isosbestic point at $1.88 \mathrm{eV}$. In situ electrochemical conductance measurements suggest that this species is responsible for modulating the bulk electrical conductivity of ProDOT(OE 3$)$-DMP. The conductance peaks with the maximal absorbance at 1.31 $\mathrm{eV}(\mathrm{ca} .+0.4 \mathrm{~V})$, and the conductance begins to drop at potentials where this spectral feature decreases in intensity (SI Figure S9). This result suggests that the charged state absorbing at 1.31 $\mathrm{eV}$ corresponds to the primary mobile carriers in the system. Therefore, we attribute the state at $1.31 \mathrm{eV}$ to mobile polarons formed upon electrochemical doping. 
For a limited range of potentials (below $+0.3 \mathrm{~V}$ ), the influence of this polaron feature does not obfuscate the structure of the neutral excitonic transition significantly. Therefore, within this potential window, we can evaluate the evolution of the main vibronic features $A_{0-0}$ and $A_{0-1}$ in terms of their energy and relative intensities. First, the energetic difference between the two principal vibronic features $\left(E_{v i b}=E_{0-0}-E_{0-1}\right)$ decreases by a factor of $\sim 0.8$, indicating a relaxation of the $\mathrm{C}=\mathrm{C}$ double bond in the polymer backbone. In addition, the exciton absorbance spectrum blueshifts considerably throughout the doping process, with the energy of the main $\mathrm{A}_{0-1}$ transition increasing by approximately $\sim 63 \pm 6 \mathrm{meV}$ (SI Figure S10). These results suggest a net disruption of the macromolecular structure and conformation of the neutral chains giving rise to the exciton population. Moreover, the absorbance ratio between the main two vibronic peaks $\left(A_{0-0} / A_{0-1}\right)$ increases by a factor of $\sim 1.7$ between -0.4 and $+0.2 \mathrm{~V}$. A similar evolution of vibronic features has been observed for chemically and electrochemically doped semicrystalline P3HT, ${ }^{28,49,50}$ where the lineshape of the UV-Vis absorption spectrum may be used as a diagnostic proxy for studying the nature of $\mathrm{HJ}$ aggregation in the film. ${ }^{51,52}$ Thomas et al. attributed the increasing $A_{0-0} / A_{0-1}$ ratio in electrochemically-gated P3HT to the preferential doping of $\mathrm{H}$-aggregates, ${ }^{28}$ which was later supported by similar studies by Harris et al. that described conformational relaxations associated with heterogeneous electrochemical doping. ${ }^{49}$ Such proposed physical pictures are consistent with previously mentioned work hypothesizing the presence of electrically resolvable chromophore populations with different effective conjugation lengths that are sequentially and selectively pdoped with increasing potential. ${ }^{46}$ In the case of ProDOT( $\left(\mathrm{OE}_{3}\right)$-DMP, we consider the additional possibility that the observed changes in the neutral lineshape during the first redox process could also be explained by distortions in chain conformation due to ion intercalation or interactions of excitons with nascent polarons. Even in the case of preferential doping of specific film domains or regions, electrolyte uptake within and around doped regions may influence the conformation of neighboring neutral domains, resulting in notable changes in the absorption lineshape. In other words, chain segments that are not oxidized during this first redox process do not necessarily remain unaffected by electrolyte uptake and charge formation occurring elsewhere in the film.

The second oxidation process in the $\mathrm{CV}$ occurs between approximately $+0.3 \mathrm{~V}$ to $+0.5 \mathrm{~V}$ (Figure 3, Region III, highlighted in yellow). During this process, the neutral state absorption continues to blue shift and loses its fine structure, suggesting the remaining neutral chromophores 
become mostly uncoupled at higher doping levels, and see an increase in inhomogeneous disorder width of the neutral exciton. This potential window also corresponds to a loss of the polaronic absorption at $1.31 \mathrm{eV}$ and the emergence of another feature further into the NIR with an isosbestic point around $1.15 \mathrm{eV}$. This low-energy feature is often attributed to bipolarons in the conventional charge carrier model. ${ }^{53,54}$ Above $+0.6 \mathrm{~V}$ (Figure 3, Region IV, highlighted in red), the neutral state absorption is completely depleted, while the $1.31 \mathrm{eV}$ polaron feature continues to decrease in intensity up to the anodic switching potential $(+0.8 \mathrm{~V})$. Upon reversing the direction of the potential sweep, the neutral state of the polymer is fully recovered. The amount of charge extracted during the oxidation process $\left(2.2 \mathrm{mC} \mathrm{cm}^{-2}\right)$ corresponds to the charge inserted during the reduction process, which provides further evidence that the doping process is repeatable.

The evolution of the linear absorption spectra with electrochemical potential raises several points of interest. (i) The lineshape of the neutral state absorption undergoes significant changes even before any substantial redox current is detected and prior to the emergence of polaronic signatures at $1.31 \mathrm{eV}$. (ii) The spectral features at $1.58 \mathrm{eV}$ and $1.11 \mathrm{eV}$ do not share the same potential dependence as the feature at $1.31 \mathrm{eV}$, which we attribute to polarons. In particular, the state at $1.58 \mathrm{eV}$ gains in strength before the appearance of the polaronic optical signature and before substantial current extraction. Moreover, the state at $1.58 \mathrm{eV}$ appears to be dependent on the properties and polarity of the surrounding environment. This observation suggests the features at 1.58 and $1.11 \mathrm{eV}$ are representative of a precursor species that mediates the electrochemical doping process and precedes polaron formation. As we will show later, the sub-gap state at $1.58 \mathrm{eV}$ is consistent with a CT-like transition. Therefore, we will refer to it as such in later discussion. (iii) The increase in $A_{0-0} / A_{0-1}$ and decrease in $E_{v i b}$ of the exciton upon doping suggests that polaron formation influences the electronic configuration of nearby neutral chains. However, linear absorption data alone cannot explain the mechanism of this influence, nor can it describe how the precursor state affects the rest of the doping reaction. Accordingly, we use resonance Raman and transient absorption spectroscopy to gain more insight into the electronic and structural coupling of doped and neural chromophores and evaluate how this coupling evolves at high doping levels. 


\section{Resonance Raman Spectroscopy}

To confirm that the NIR state indeed constitutes an electronic transition that is distinct from the neutral exciton and is coupled to a unique molecular conformation, we performed in situ resonance Raman spectroscopy utilizing two distinct excitation energies. Raman spectroscopy is a very sensitive probe of the local environment and detects vibrational modes that show a change in the molecular polarizability upon vibration. ${ }^{55}$ In particular, resonance Raman spectroscopy enhances modes coupled to the specific electronic transition in resonance with the laser excitation. This enhancement occurs over orders of magnitude and assists in identifying vibrational modes that are uniquely coupled to distinct electronic transitions. For evaluating films of ProDOT(OE3)-DMP, we employed two excitation energies: one in resonance with the $\pi$ - $\pi^{*}$ electronic transition of neutral chains $(2.33 \mathrm{eV})$ and a second excitation resonant with the sub-gap transition at $1.58 \mathrm{eV} .{ }^{56}$ 
A.
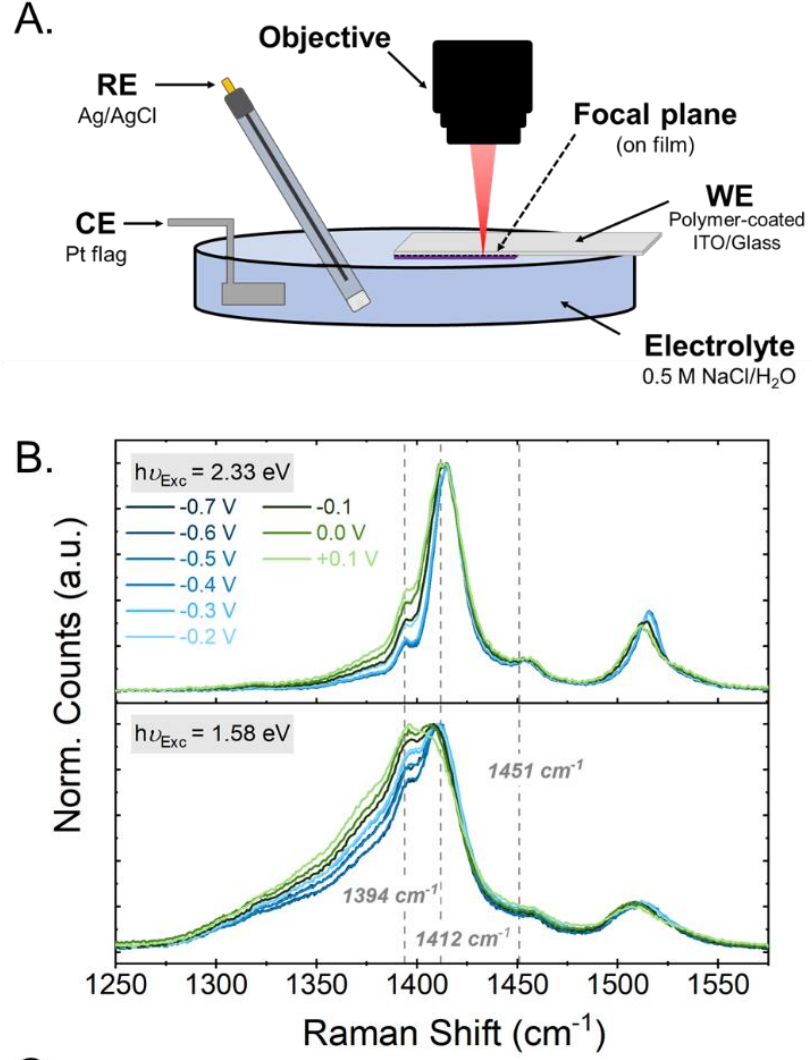

C.

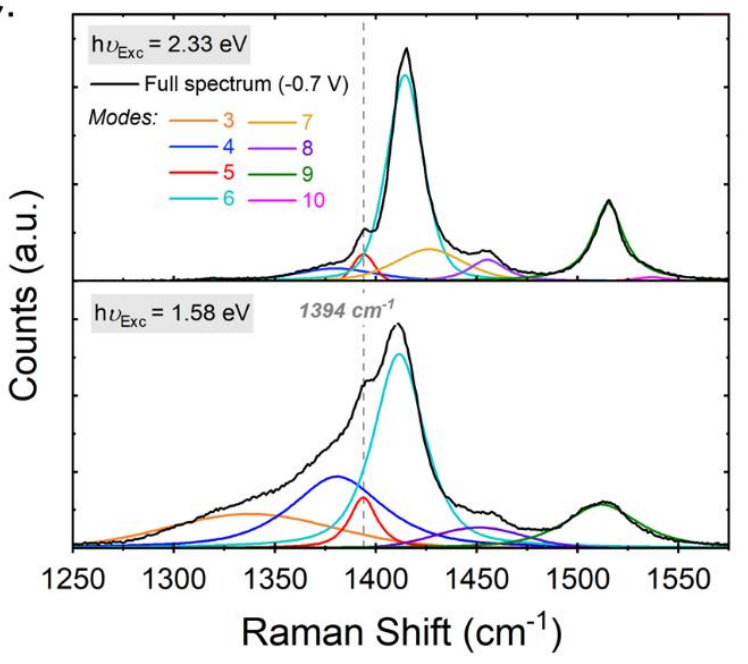

Figure 4: (A) Schematic of cell used for in situ Raman measurements performed in an aqueous electrolyte, showing working, reference, and counter electrode (WE, RE, and CE, respectively) and confocal objective. (B) Normalized potential-dependent Raman spectra of ProDOT(OE $)_{3}$ DMP using an excitation energy of $2.33 \mathrm{eV}$, which is resonant with the $\pi-\pi^{*}$ transition of the neutral, de-doped polymer (top), and an excitation energy of $1.58 \mathrm{eV}$, which is resonant with the 
sub-gap transition (bottom). Spectra were collected in situ while biasing the polymer film at the potentials indicated by the color legend. (C) Peak deconvolution for a select region of the Raman spectrum of ProDOT(OE 3 -DMP at $-0.7 \mathrm{~V}$ for an excitation energy of $2.33 \mathrm{eV}$ (top) and an excitation energy of $1.58 \mathrm{eV}$ (bottom). Data is shown as a black line with deconvoluted modes superimposed as colored lines and labeled as in Table 1. All data was collected in a $0.5 \mathrm{M}$ $\mathrm{NaCl} / \mathrm{H}_{2} \mathrm{O}$ electrolyte, and potentials are reported $v$ s. $\mathrm{Ag} / \mathrm{AgCl}$.

When the polymer is in its charge-neutral state at $-0.7 \mathrm{~V}$, the most intense mode for both excitations is at $\sim 1412 \mathrm{~cm}^{-1}$ (SI Figure S11), which corresponds to the symmetric stretching of the $\mathrm{C}_{\alpha}=\mathrm{C}_{\beta}$ double bond in neutral chains. ${ }^{28}$ When exciting at $1.58 \mathrm{eV}$, we note a relative enhancement of the modes at $\sim 1338 \mathrm{~cm}^{-1}, \sim 1381 \mathrm{~cm}^{-1}$, and $\sim 1394 \mathrm{~cm}^{-1}$ (labeled modes 3,4 , and 5 in Table 1). This indicates that these three modes are in resonance with the CT-like transition but not with the neutral electronic transition. This observation establishes that the spectral feature at $1.58 \mathrm{eV}$ indeed corresponds to a distinct electronic transition coupled to a unique molecular geometry. The potential energy surface (PES) of any molecular conformation is determined by the linear combination of all the vibrational modes coupled to it: the PES associated with the CT transition will then be distinct with respect to the PES associated to the $\pi$ - $\pi^{*}$ transition and will have as part of the basis set the modes at frequencies $\sim 1338 \mathrm{~cm}^{-1}, \sim 1381 \mathrm{~cm}^{-1}$, and $\sim 1394 \mathrm{~cm}^{-1}$. In other words, the electron distribution and the normal vibrational modes associated with the CT transition are distinct from the ones associated with the neutral configuration. To assign the Raman modes associated with ProDOT(OE 3$)$-DMP (Table 1), we adapt our interpretation from a thorough set of assignments made previously for poly(3,4-ethylenedioxythiophene) (PEDOT), a structurallysimilar poly(dioxythiophene) ${ }^{57,58}$ Based on these interpretations, the enhanced vibration at $\sim 1394$ $\mathrm{cm}^{-1}$ can be assigned to the $\mathrm{C}_{\beta}-\mathrm{C}_{\beta}$ stretching mode of the thiophene heterocycles. Previous resonance Raman spectroscopy studies on P3HT associated an increase in the intensity ratio of the $\mathrm{C}_{\beta}-\mathrm{C}_{\beta} / \mathrm{C}_{\alpha}=\mathrm{C}_{\beta}$ stretching modes with enhanced chain planarity in annealed P3HT samples. ${ }^{59,60}$ Because the $\mathrm{C}_{\beta}-\mathrm{C}_{\beta}$ mode is enhanced when using an excitation resonant with the CT-like state at $1.58 \mathrm{eV}$, we hypothesize that this electronic transition is preferentially associated with chromophore segments with enhanced intramolecular ordering and planarity. When evaluating the potential dependence of the Raman spectrum excited with $1.58 \mathrm{eV}$ laser (Figures 4A and 4B), we 
note that the modes coupled to the CT-state (modes 3, 4, and 5) increase in their relative intensity between $-0.6 \mathrm{~V}$ and $-0.3 \mathrm{~V}$ (SI Figures S13, S17): the changes in the Raman modes coupled to the CT-state occur prior to electrochemical doping and before the emergence of the polaronic signature $(1.31 \mathrm{eV}$ ) at $\sim 0.1 \mathrm{~V}$ in the linear spectra (Figure 2B). The other modes in the $1.58 \mathrm{eV}$ excitation Raman spectrum do not change considerably before the oxidation onset at ca. -0.2 V (SI Figures S13). Similar changes can be observed in the $2.33 \mathrm{eV}$ excitation spectra (Figure 4A), where relative intensity of mode 5 increases prior to the oxidation onset (SI Figures S12, S15). While these changes are less significant than those observed for the $1.58 \mathrm{eV}$ excitation spectra (Figure 4B), these observations are consistent with a modulation of the ground state geometry preceding electrochemical doping. As described above, previous characterization of the Raman modes of polythiophenes suggests that the ground state modulation identified here for ProDOT(OE 3$)-\mathrm{DMP}$ may be associated with planarization of the thiophene backbone.

Based on these data, the CT-like state at $1.58 \mathrm{eV}$ appears to either passively mediate the electrochemical doping process in ProDOT(OE3)-DMP or it provides a driving force toward polaron formation by favorably modulating of the PES in a manner that facilitates charge carrier formation. While neither possibility can currently be excluded, as we are unable to distinguish spectator modes from active modes in the Raman spectra, we hypothesize that the CT transition provides a driving force for electrochemical doping. Seeing as the initial stages of doping are also accompanied an increase in the relative intensity of mode 5 (and therefore may also be thought to be associated with increased chain planarity, based on previous literature ${ }^{59,60}$ ), we hypothesize that the CT-like transition facilitates doping by enhancing the level of intramolecular order in the polymer, thereby offering greater conformational stabilization to nascent charge carriers. 
Table 1: Raman modes of ProDOT(OE $\left.\mathrm{OE}_{3}\right)-\mathrm{DMP}$ films spray-cast onto ITO/glass substrates and immersed in $0.5 \mathrm{M} \mathrm{NaCl} / \mathrm{H}_{2} \mathrm{O}$, applying $-0.7 \mathrm{~V}$ vs. $\mathrm{Ag} / \mathrm{AgCl}$. Mode assignment is based on Raman modes of PEDOT, as reported in the literature. ${ }^{57}$

\begin{tabular}{|c|c|c|c|c|}
\hline $\begin{array}{c}\text { Mode } \\
\quad \#\end{array}$ & $\begin{array}{c}\text { Peak Position } \\
\text { for } \\
2.33 \text { eV Excitation } \\
\left(\mathrm{cm}^{-1}\right)\end{array}$ & $\begin{array}{c}\text { Peak Position } \\
\text { for } \\
1.58 \mathrm{eV} \text { Excitation } \\
\left(\mathrm{cm}^{-1}\right)\end{array}$ & $\begin{array}{c}\text { Literature } \\
\text { Peak Position for } \\
1.58 \text { eV Excitation } \\
\left(\mathrm{cm}^{-1}\right)\end{array}$ & $\begin{array}{c}\text { Mode } \\
\text { Assignment }\end{array}$ \\
\hline 1 & 1103 & 1085 & 1097 & $\begin{array}{c}\text { C-O-C } \\
\text { deformation }\end{array}$ \\
\hline $2^{a}$ & 1205,1217 & 1178,1208 & 1252 & $\begin{array}{l}\mathrm{C}_{\alpha}-\mathrm{C}_{\alpha} \text { (inter- } \\
\text { ring) stretching }\end{array}$ \\
\hline $\mathbf{3}^{\mathbf{b}}$ & 1320 & 1337 & - & - \\
\hline $4^{b}$ & 1380 & 1381 & - & \\
\hline $5^{b}$ & 1394 & 1394 & 1370 & $\mathrm{C}_{\beta}-\mathrm{C}_{\beta}$ stretching \\
\hline $\mathbf{6}^{\mathbf{b}}$ & 1414 & 1412 & 1414 & $\begin{array}{l}\mathrm{C}_{\alpha}=\mathrm{C}_{\beta}(-\mathrm{O}) \\
\text { symmetric } \\
\text { stretching }\end{array}$ \\
\hline 7 & 1426 & - & - & - \\
\hline $8^{c}$ & 1455 & 1451 & 1445 & $\begin{array}{c}\mathrm{C}_{\alpha}=\mathrm{C}_{\beta}(-\mathrm{O}) \\
\text { symmetric } \\
\text { stretching of } \\
\text { doped segments } \\
\text { in PEDOT }\end{array}$ \\
\hline 9 & 1515 & 1512 & 1516 & $\begin{array}{l}\mathrm{C}_{\alpha}=\mathrm{C}_{\beta}(-\mathrm{O}) \\
\text { asymmetric } \\
\text { stretching }\end{array}$ \\
\hline 10 & 1536 & - & - & - \\
\hline
\end{tabular}


${ }^{a}$ At $-0.7 \mathrm{~V}$, this mode is represented by a single peak in the $2.33 \mathrm{eV}$ excitation spectrum, but appears as two overlapping peaks in the $1.58 \mathrm{eV}$ excitation spectrum. With increasing doping level, the single peak in the $2.33 \mathrm{eV}$ excitation spectrum splits into two distinct signals whose relative intensity varies with potential.

${ }^{\mathrm{b}}$ Raman modes of ProDOT( $\left(\mathrm{OE}_{3}\right)$-DMP that appear to be coupled to the CT-like transition at 1.58 $\mathrm{eV}$.

${ }^{\mathrm{c}}$ Previous work documenting the Raman modes of PEDOT has ascribed this mode to the $\mathrm{C}_{\alpha}=\mathrm{C}_{\beta}(-$ O) symmetric stretching of doped segments. ${ }^{57}$ For electrochemically doped ProDOT( $\left.\mathrm{OE}_{3}\right)$-DMP, there is not a substantial increase in the intensity of this mode with increasing doping level. In fact, the relative contribution of this mode becomes insignificant at high potentials/doping levels (SI Figures S12, S13. Accordingly, we do not find this mode to be associated with doped chromophores and therefore leave it unassigned for ProDOT( $\left.\mathrm{OE}_{3}\right)-\mathrm{DMP}$.

For both excitation energies (2.33 and $1.58 \mathrm{eV})$, Raman mode $6\left(\sim 1414 \mathrm{~cm}^{-1}\right.$, corresponding to the $\mathrm{C}_{\alpha}=\mathrm{C}_{\beta}(-\mathrm{O})$ symmetric stretch) does not exhibit any significant change until after the onset of oxidation at $-0.21 \mathrm{~V}$ vs. $\mathrm{Ag} / \mathrm{AgCl}$. This mode red-shifts during the first redox process (SI Figures S14, S16), representing a relaxation of the $\mathrm{C}_{\alpha}=\mathrm{C}_{\beta}(-\mathrm{O})$ bond as it adopts a more single-bond character. In P3HT films, a shifting of this mode to lower frequencies has been attributed to greater torsional order and enhanced delocalization of $\pi$-electron, which again suggests that the early stages of doping are characterized by enhanced chain planarity. ${ }^{59-61}$ Similarly, mode $9\left(\sim 1515 \mathrm{~cm}^{-}\right.$ ${ }^{1}$, corresponding to the $\mathrm{C}_{\alpha}=\mathrm{C}_{\beta}(-\mathrm{O})$ asymmetric stretch) also red shifts upon the initial oxidation of the polymer and then blue-shifts after the onset of the second redox process (SI Figure S12, S13). At potentials spanning the second oxidation process $(-0.1$ to $+0.2 \mathrm{~V})$, mode $5\left(1394 \mathrm{~cm}^{-1}\right.$, corresponding to the $\mathrm{C}_{\beta}-\mathrm{C}_{\beta}$ stretch) first red-shifts, indicating a relaxation of the $\mathrm{C}_{\beta}-\mathrm{C}_{\beta}$ bond. After $+0.4 \mathrm{~V}$ and during the second redox process, this mode shifts to higher energies (SI Figures S14, S16) representing a stiffening of the $\mathrm{C}_{\beta}-\mathrm{C}_{\beta}$ bond and a return to its original more double bond character. The blue-shifting of this mode at $+0.4 \mathrm{~V}$ coincides with the same potential associated with a decrease in intensity of polaronic absorbance at $1.31 \mathrm{eV}$, the decrease in electrochemical conductance, and the emergency of the low-energy bipolaron signature further into the NIR. Together, these results indicate that during the early stages of doping and charge carrier formation, 
the chain segments rearrange themselves, resulting in stiffer inter-ring bonds, relaxed intra-ring bonds, which is indicative of greater chain planarity and torsional order. Meanwhile, the formation of bipolarons stiffens the intra-ring bond. In view of our observations, we can revisit the points raised at the end of our analysis of the linear absorption spectra. Point $(i)$, which concerns changes in the linear absorption occurring prior to any substantial redox current and the appearance of the optical signature of polarons, can be explained by a polarization of the $\pi$-electrons in the polymer, which results in a modulation of the energy of the vibrational modes coupled to the neutral electronic transition. This manifests in changes in the resonance Raman spectra, which are similarly observed prior to the oxidation onset of the polymer. The blue-shift of the $\mathrm{C}_{\alpha}=\mathrm{C}_{\beta}(-\mathrm{O})$ symmetric stretch we observe in the Raman spectra upon doping is also consistent with the reduction in $\mathrm{E}_{\mathrm{vib}}$ in the linear absorption spectra, most likely due to swelling with electrolyte as counter ions intercalate into the film to support electronic charges on the polymer backbone. Points (ii) and (iii), which concern the unique potential-dependence of the $1.58 \mathrm{eV}$ state and the apparent influence of charged states on remaining neutral species, are further refined. From the resonance Raman studies, we observe distinct changes in the PES of the polymer for different electrochemical processes. The formation of the polaron feature is associated with a stiffening of the neutral chains and increased overall chain planarity, while further oxidation of the backbone to bipolaronic states stiffens the $\mathrm{C}_{\alpha}-\mathrm{C}_{\alpha}$ intra-ring bonds as well as the inter-ring bonds. It is interesting to look at the in situ conductance in light of this observation (SI Figure S9): it is not until the second oxidation process $($ ca. $+0.3 \mathrm{~V})$ that there is a steep increase in the conductance arising from doping. Mode 2 (the $\mathrm{C}_{\alpha}=\mathrm{C}_{\beta}(-\mathrm{O})$ symmetric stretch) therefore seems to be associated with the conductivity onset, while the other modes do not show such an association with the conductivity modulation. At higher potentials (above $+0.5 \mathrm{~V}$ ), the conductance then decreases: this corresponds to the potential range where we observe the loss of structure and depletion of the neutral species in the linear absorption data (Figure 3A, Regions III and IV).

\section{In Situ Transient Absorption Spectroscopy}

As detailed above, the Raman spectroscopy results confirmed the distinct nature of the precursor state at $1.58 \mathrm{eV}$ with respect to the neutral transition, and the potential-dependence of the Raman spectra shed light on the role of this state in the doping process. To address the 
formation and evolution of this state at $1.58 \mathrm{eV}$ and its coupling to the neutral excitation, we next performed transient absorption (TA) spectroscopy in situ while electrochemically doping ProDOT( $\left(\mathrm{OE}_{3}\right)$-DMP in the same electrolyte system, as shown in Figure 5A.

TA spectroscopy probes electronic states and their dynamics following photoexcitation. In a typical experiment, a spectrally broad laser pulse is used to probe the changes caused by a second pulse in the optical response of the sample. This second pulse, called the pump beam, is spectrally narrower and temporally delayed with respect to the first pulse, the probe beam. The recorded signal $\Delta T / T=\left(T_{\text {pumpON }}-T_{\text {pumpOFF }}\right) / T_{\text {pumpOFF }}$ is a function of energy (i.e. inverse wavelength within a factor of $h c$, where $h$ is the Planck constant and $c$ is the speed of light in vacuum) and corresponds to the relative change in the transmission of the probe induced by the pump, for a fixed time delay. Figures 5B-C compare the features of the linear absorption and TA spectra for films of ProDOT(OE 3 )-DMP held at the same potential (-0.7 V vs. Ag/AgCl). In Figure 5B, the exemplary TA data was collected using a pump energy of $2.29 \mathrm{eV}$, which matches the absorption of the neutral, de-doped polymer chains.

Depending on the energy of the probe pulse, three different scenarios are possible: ${ }^{62}$ (i) The probe energy is resonant with the electronic transition $S_{0} \rightarrow S_{n}$ (ground state absorption). In this situation, the pump depletes the electronic ground state and the transmission of the sample is greater than for the unperturbed system (positive $\Delta T / T$ signal), giving rise to a photo-bleaching (PB) signature. In general, PB spectrally overlaps with the linear absorption. In the case of ProDOT( $\left(\mathrm{OE}_{3}\right)$-DMP, the $\mathrm{PB}$ extends from $1.85 \mathrm{eV}$ to $\sim 2.48 \mathrm{eV}$ and bears a similar lineshape as the linear absorption (Figures 5B-C). (ii) The probe energy is resonant with transitions $S_{n} \rightarrow S_{n+1}$. Here, the pump populates these upper excited states; hence $\Delta T / T$ is a negative quantity, which is associated with a photo-induced absorption (PA) signal. In neat, pristine $\pi$-conjugated materials, the PA signal is usually assigned to the lowest singlet $S_{l}$ excitons. ${ }^{62}$ In Figure 5C, PA extends for energies below $1.85 \mathrm{eV}$, with a notable depression at $\sim 1.58 \mathrm{eV}$ which, as will be elaborated on later, we interpret as a localized PB superimposed on a PA band. (iii) The probe pulse is in resonance with the transition $S_{1} \rightarrow S_{0}$. If this transition is optically allowed, the probe can induce stimulated emission (SE) from the level $S_{l}$ to the ground state, which will be perceived as an increase in transmission (positive $\Delta T / T$ signal). The energies of the SE signal typically correspond to those associated with the photoluminescence spectrum. In the case of ProDOT( $\left.\mathrm{OE}_{3}\right)-\mathrm{DMP}$, this 
transition is either forbidden or very weak, so we do not see this signal in our data. By changing the temporal delay between pump and probe beams, it is possible to reconstruct the temporal dynamics of the PB and PA, as shown in Figure for time delays of 0.6 ps, 10 ps, 100 ps and 900 ps.

A.

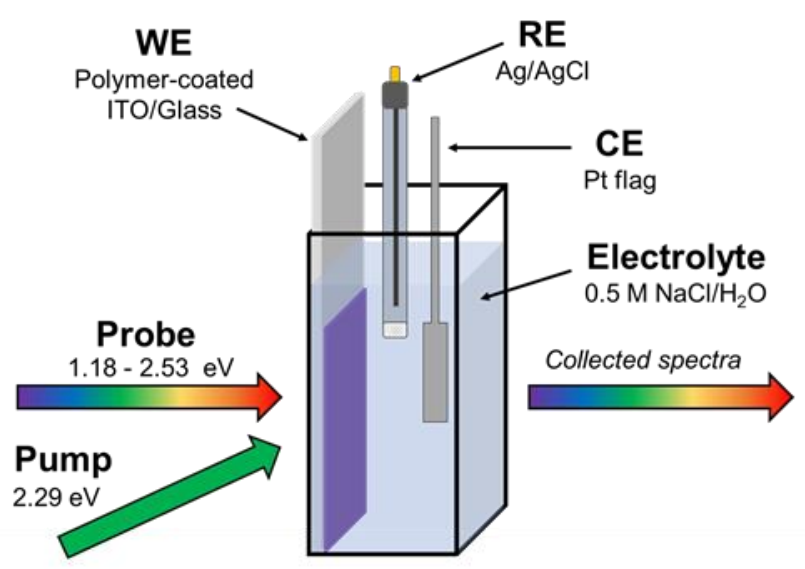

B.

$$
\text { Energy (eV) }
$$

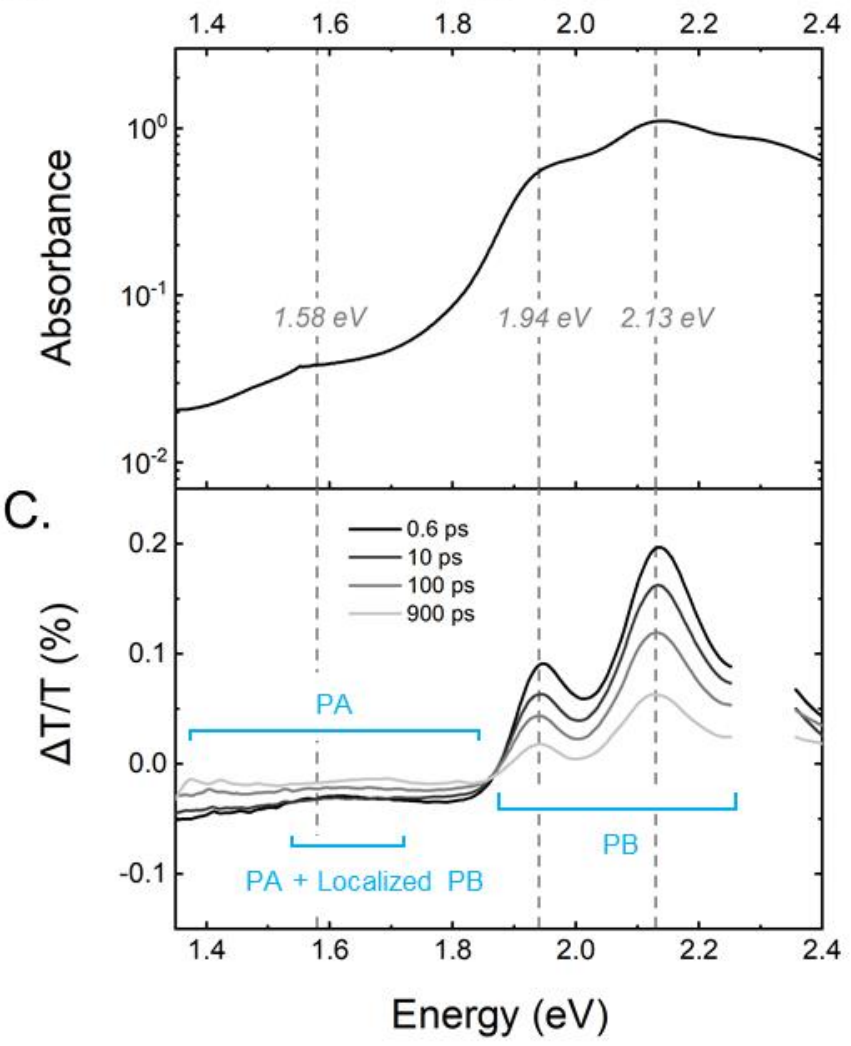


Figure 5: (A) Schematic of the cell used for in situ transient absorption measurements performed in an aqueous electrolyte, showing working, reference, and counter electrode (WE, RE, and CE, respectively) and pump/probe pulses. (B) Linear absorbance spectrum of ProDOT(OE 3 )-DMP at $0.7 \mathrm{~V}$ vs. $\mathrm{Ag} / \mathrm{AgCl}$ in $0.5 \mathrm{M} \mathrm{NaCl} / \mathrm{H}_{2} \mathrm{O}$, highlighting the sub-gap absorbance feature at $\sim 1.58 \mathrm{eV}$ and the vibronic progression of the primary photoexcitation $\left(A_{0-0}\right.$ at $1.94 \mathrm{eV}, A_{0-1}$ at $\left.2.13 \mathrm{eV}\right) .(\mathbf{C})$ Transient absorption spectra of ProDOT( $\left(\mathrm{OE}_{3}\right)$-DMP at $-0.7 \mathrm{~V}$ for a pump-probe time delays of 0.6 ps, 10 ps, 100 ps, and 900 ps respectively. For clarity, data corresponding to pump energy (2.29 $\mathrm{eV})$ are removed. Regions labeled in blue brackets and text correspond to the photobleach of the primary photoexcitation (PB), the photoinduced absorption (PA), and the localized PB superimposed on the PA occurring at $\sim 1.58 \mathrm{eV}$.

We will first consider the region corresponding to the PA signal $(0.95 \mathrm{eV}-1.85 \mathrm{eV})$. Figures 6A-B compare the linear absorption spectra and TA spectra recorded in situ for ProDOT(OE 3$)$ DMP across a series of electrochemical potentials. The TA spectra presented in Figure 6B correspond to a pump-probe delay of $0.6 \mathrm{ps}$. To highlight the changes in the spectral features, data are shown as absolute values on a logarithmic scale. During the first oxidation process (Figure 2, Region II) the PA band arising from the lowest $S_{1}$ exciton is quenched (Figure 6B and SI Figure $\mathrm{S} 19$ ) and the depression at $\sim 1.58 \mathrm{eV}$ becomes more pronounced with increasing doping level. Because this energy matches the spectral feature at $1.58 \mathrm{eV}$ in the linear absorption data, we interpret this depression as a localized PB signal superimposed to the broader PA band. Such assignment is also illustrated for a single potential (-0.7 V) in Figure 5B, for clarity. This localized signal appears soon after the pump interaction (resolution limited $\sim 220 \mathrm{fs}$ ), hence it appears to be a common ground state with the neutral exciton (SI Figure 18). 

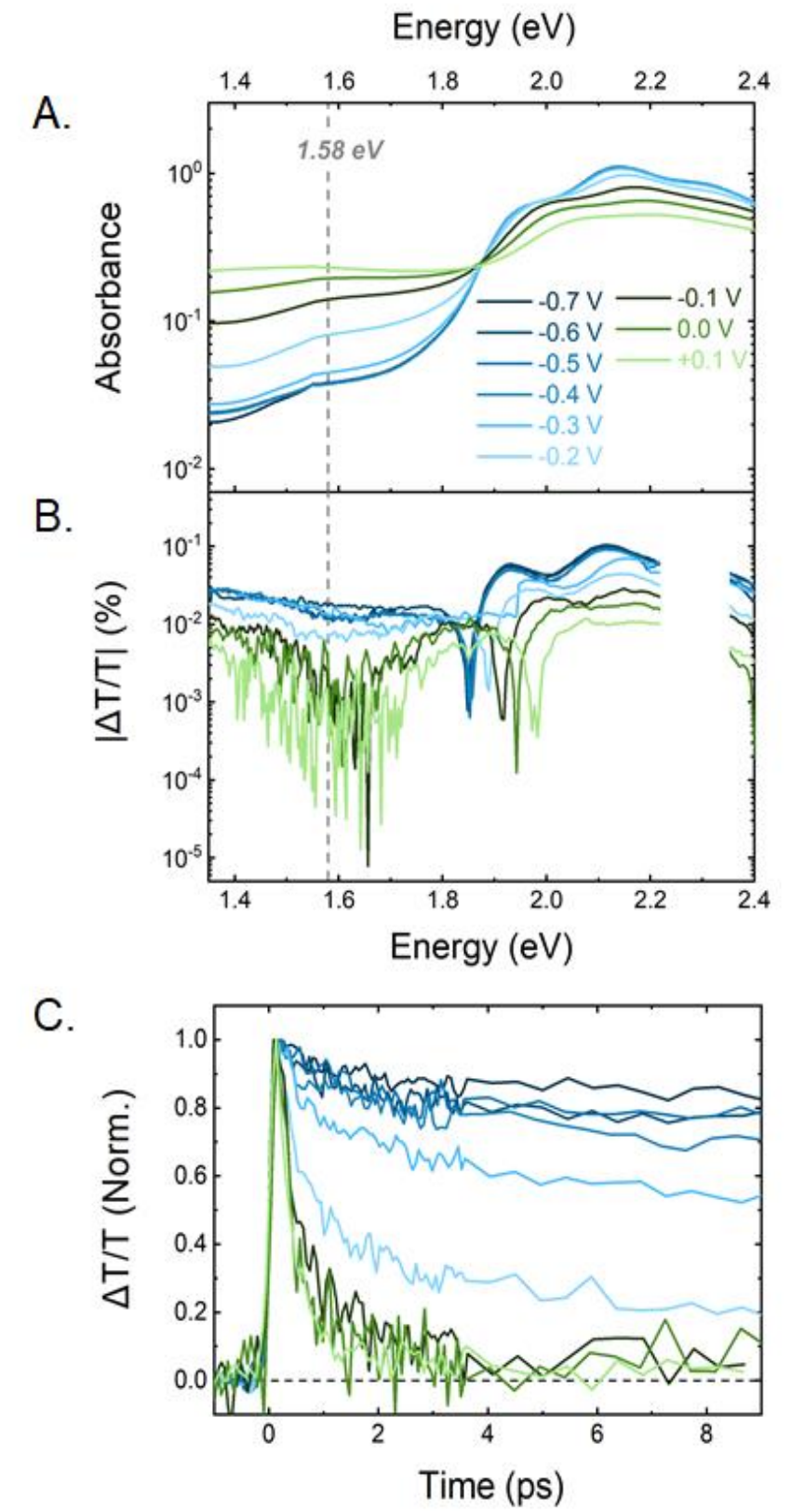

Figure 6: (A) Linear absorption spectra of ProDOT $\left(\mathrm{OE}_{3}\right)-\mathrm{DMP}$ as a function of potential vs. $\mathrm{Ag} / \mathrm{AgCl}$ in a $0.5 \mathrm{M} \mathrm{NaCl} / \mathrm{H}_{2} \mathrm{O}$ electrolyte. From dark blue to light green curves: $-0.7 \mathrm{~V}$ to $+0.1 \mathrm{~V}$ in even steps of $0.1 \mathrm{~V}$. (B) Transient absorption spectra of ProDOT(OE 3 -DMP as a function of potential vs. $\mathrm{Ag} / \mathrm{AgCl}$, using identical labeling convention as 4A. Data correspond to a pumpprobe delay of $0.6 \mathrm{ps}$. For clarity, data spanning the pump energy $(2.29 \mathrm{eV})$ are removed. (C) 
Dynamics of the ground state bleach (probe energy $=2.14 \mathrm{eV}$ ) from $-0.7 \mathrm{~V}$ to $+0.1 \mathrm{~V}$ vs. $\mathrm{Ag} / \mathrm{AgCl}$ (in steps of $0.1 \mathrm{~V}$ ) in a $0.5 \mathrm{M} \mathrm{NaCl} / \mathrm{H}_{2} \mathrm{O}$ electrolyte, using the coloring convention in $4 \mathrm{~A}$.

Next, we examine the PB of ProDOT( $\left(\mathrm{OE}_{3}\right)$-DMP and its evolution with doping level. We observe that starting from $-0.3 \mathrm{~V}$ (prior to the current onset in the $\mathrm{CV}$ shown in Figure $2 \mathrm{~A}$ ), the intensity ratio between the two main peaks identified as $A_{0-0}$ and $A_{0-1}(\sim 1.92 \mathrm{eV}$ and $\sim 2.10 \mathrm{eV})$ increases. Meanwhile, $E_{0-0}$ blue-shifts as the potential increases, while the spacing between these two peaks $E_{\text {vib }}^{\prime}$ decreases. These changes in the TA line shape are very similar to those we see in the linear absorption data, even though there is a slight discrepancy in the absolute values (SI Figure S10). This observation confirms that the potential dependence of $A_{0-0} / A_{0-1}, E_{0-0}$, and $E^{\prime}{ }_{v i b}$ is not an artifact arising from interference from the polaron band tail. While the change in the lineshape of the PB is similar to that observed in the linear absorption data, the reduction in its intensity is evidence of a common ground state between the exciton population and the state at $1.58 \mathrm{eV}$, since the exciton population undergoes ultrafast quenching ( $<230 \mathrm{fs}$, resolution limited) even though the pump excitation densities were progressively increased to compensate for the loss in absorption. For potentials greater than $-0.3 \mathrm{~V}$, exciton dynamics become increasingly faster (Figure 6C), especially after we reach the potential corresponding to the onset of redox current. We can attribute this behavior to processes of exciton-charge annihilation ${ }^{25}$ that appears even at the low fluences used here by virtue of the high charge density introduced during electrochemical doping.

Similar results were obtained when the measurements were repeated under an inert atmosphere using a degassed aqueous electrolyte held under an argon blanket during TA measurements (SI Figure S20, S21). Although dynamics are faster in the presence of oxygen, data collected in the degassed environment still show the PB at $1.58 \mathrm{eV}$ superimposed to the PA signature. In addition, an analogous trend is observed in the potential dependence of the PB dynamics. While these results suggest that environmental oxygen does act as a moderate quenching agent with respect to the $\mathrm{PB}$, they also demonstrate that oxygen does not play a substantial role in the formation of the CT-like state at $1.58 \mathrm{eV}$, nor does it drastically change the doping process. For comparison, TA experiments were performed using ProDOT( $\left.\mathrm{OE}_{3}\right)$-DMP in a polar aprotic electrolyte solution of $\mathrm{TBAPF}_{6}$ in propylene carbonate (SI Figures S22, S23). While the $1.58 \mathrm{eV}$ state is still present in 
the de-doped state, the dynamics of the $\mathrm{PB}$ recovery are much faster in $\mathrm{TBAPF}_{6} / \mathrm{PC}$ and the potential dependence of the quenching is less pronounced when compared to the dynamics observed in $\mathrm{NaCl} / \mathrm{H}_{2} \mathrm{O}$. The increased polarity of the aqueous electrolyte and the differences in the charge density evolution for each system may account for the observed differences, as will be explained in the following section.

\section{Discussion}

Based on the emergence of the state at $1.58 \mathrm{eV}$ prior to any substantial redox current and polaron formation in the linear absorption, we exclude the possibility that this state represents a polaronic species in the traditional sense. Simultaneously, these results suggest that the state is a precursor to full polaron formation during the doping process. Resonance Raman spectra prove that this state represents an electronic transition that is distinct from the neutral species, as it is coupled to a unique set of vibrational modes. Insights from the transient absorption (TA) spectra show that this species corresponds to a narrow photobleach (PB) that appears contextually to the PB of the neutral transition, indicating that these two states share a common ground state. In sum, these observations lead us to conclude that the precursor state observed at $1.58 \mathrm{eV}$ represents a polarized molecular excitation, i.e. a fundamental molecular excitation which has acquired a charge-transfer character. For this reason, it has been denoted as a charge transfer state (CT-state). This assignment is in agreement with a previous study on the photo-physics of P3HT nanoparticles (NPs) in aqueous buffers. ${ }^{63}$ In the case of P3HT NPs dispersed in ultrapure water, the linear absorption showed a spectral component at low energies $(\sim 1.8 \mathrm{eV})$ that is not present or appreciable in thin films of the same material. This component was enhanced in acidic aqueous buffers ( $\mathrm{pH}$ values from 3 to 11 were tested). The effect was suppressed for encapsulated NPs, where a lipid-PEG corona was used to shield the NPs from the buffer. TA measurements showed a PB band corresponding to the $1.8 \mathrm{eV}$ feature for NPs in acidic buffers $(\mathrm{pH}=3)$. In this study, these observations were rationalized by assigning the low energy feature to a distribution of CT states due to polarization of $\pi$-electrons in the polythiophene backbone. This polarization is enhanced at low $\mathrm{pH}$, where there are more coulombic interactions between backbone and ionic species, and complexes are formed with electron-withdrawing species that are thought to serve as a precursor for charge generation. Because CT states of this nature are mainly non-radiative, the 
photoluminescence measurements of these NPs were shown to be insensitive to $\mathrm{pH}$ variations. ${ }^{63}$ Here, in the case of electrochemically addressed ProDOT( $\left.\mathrm{OE}_{3}\right)-\mathrm{DMP}$, the polarization of the excitation could most likely arise from the applied bias and associated ion infiltration into the film. Notably, our TA data collected in an inert atmosphere exclude the possibility that complexation with molecular oxygen plays a central role in the process (SI Figures S20, S21).

The fact that the CT-state is coupled to a unique set of molecular vibrations implies that its electronic configuration and thus its PES are different from those of the neutral ground configuration. In the context of the doping process, this opens up two possible scenarios: on the one hand, the CT-state could play an active part by preparing the PES, favoring the doping of chain and driving the reaction; on the other hand, it could just assume a passive role and therefore not be critical to the doping.

In contrast to the findings of Tsokkou et al., who evaluated acid doping of the donor-acceptor system PCPDTBT, ${ }^{38}$ we do not observe a broad bleaching feature corresponding to the energy of the polaron (SI Figure S18). In fact, for ProDOT(OE 3$)$-DMP, we see no evidence for a shared ground state between the exciton and polaron. Even at $+0.1 \mathrm{~V}$ vs. $\mathrm{Ag} / \mathrm{AgCl}$, when there is a coexistence of both the neutral exciton $(\sim 2.13 \mathrm{eV})$ and the polaron $(\sim 1.31 \mathrm{eV})$, pumping of the exciton does not yield any apparent bleaching of the polaron, as evidenced by the undisturbed PA signal spanning into this range (SI Figures S24, S25). Our evidence suggests that there are subtle differences in the elementary steps governing charge carrier formation in conjugated polymers. These differences, which appear to depend on the mechanism of doping, the extent of doping, as well as the polymer structure, warrant further investigation to better characterize and understand charge carrier formation in conjugated polymers. A notable difference in the work conducted by Tsokkou et al. is the use of an acid to dope a donor-acceptor system which has counterions fixed to the side chains (a conjugated polyelectrolyte), ${ }^{38}$ whereas the work presented here employs electrochemical doping to address an all-donor polymer where the permeation of exogeneous counterions are necessary to stabilize charge carriers induced by the electrochemical potential. Presently, the factors governing the formation of shared ground state complexes are unclear, although differences in the frontier orbital configuration (donor-acceptor or all donor), doping mechanism (chemical, acid, or electrochemical), the presence of tethered counter ions, film microstructure, and side chain polarity (polar, apolar, or ionic) may play a substantial role. 
What seems to be common to both the acid-doped PCPDTBT ${ }^{38}$ and our analysis of electrochemically doped ProDOT(OE $\mathrm{OE}_{3}$-DMP is the effect of charge concentration on the dynamics of the PB. In both cases, doping is associated with a quenching of the PB, which is to be expected given the general phenomenon of exciton-charge annihilation. ${ }^{64}$ Tsokkou et al. reported dynamics spanning $\sim 1$ ps for the fully p-doped polyelectrolyte. ${ }^{38}$

For ProDOT( $\left(\mathrm{OE}_{3}\right)-\mathrm{DMP}$, we also note that the excited state dynamics are sensitive to the nature of the electrolyte. In a polar aprotic electrolyte $\left(0.5 \mathrm{M} \mathrm{TBAPF}_{6}\right.$ in propylene carbonate), the recovery of the ground state bleach is notably reduced, even for similar oxidation states or doping levels (SI Figure S20). There are several possible origins for this behavior. The higher dielectric constant of the aqueous medium could increase the coulombic screening of the excited state, thereby extending its lifetime. In addition, the de-doped films may also exhibit different degrees of electrolyte trapping after multiple doping/de-doping cycles. In other words, while both films may be in the same oxidation state, the surrounding ionic milieu may solvate each polymer to different extents. Given the known propensity of oligo(ether) chains to chelate small alkali metal ions such as sodium, ${ }^{65-68}$ we expected the electrochemical cycling of ProDOT(OE 3$)$-DMP in the aqueous sodium chloride electrolyte to potentially result in a greater enrichment of ions in the dedoped film, whereas fewer ions may be trapped when a polar aprotic electrolyte system comprising large polarizable ions $\left(\mathrm{TBAPF}_{6}\right.$ in $\left.\mathrm{PC}\right)$. Such dissimilarities in film solvation and ion content could both disrupt the ordering of chromophores and also change the overall dielectric quality of the environment, thereby influencing the excited state dynamics. We note that the absorbance profiles of de-doped ProDOT(OE3)-DMP appears to have distinct lineshapes depending on the surrounding electrolyte, with the film immersed in $\mathrm{NaCl} / \mathrm{H}_{2} \mathrm{O}$ exhibiting an enhanced high-energy absorbance feature (SI Figure S26). These are suggestive of subtle changes in the molecular-level arrangement and coupling of chromophores, which may also influence the stabilization and quenching of photoexcitations.

Not only are the dynamics faster in the polar aprotic electrolyte, but the potential-dependence of the recovery dynamics is also weaker when compared to the potential dependence observed in the aqueous medium (SI Figure S23). As the charge densities at the early stages of doping is similar for regardless of the electrolyte (SI Figure S27), these differences cannot be ascribed to different doping densities. One possibility is that the lower dielectric constant of the organic electrolyte 
provides a reduced Coulomb screening (in the Langevin picture), hence making the interaction between excitons and charges stronger, quenching exciton population in such an effective way in the organic electrolyte that the effect of increasing charge density is reduced. From these findings, we emphasize that the photophysical properties of OMIECs are highly dependent on not only the doping level, but also on the nature and composition of the surrounding electrolyte. The presence of unique polymer-electrolyte interactions give rise to notable differences in optical and electrochemical observables, as shown here for ProDOT( $\left(\mathrm{OE}_{3}\right)$-DMP. This suggests a particularly strong coupling of the molecular properties to the surrounding environment and highlights a need to explore the wide space of environmental parameters (protic and aprotic systems, ion size and structure, polarity, hydrogen bonding, $\mathrm{pH}$, etc.) to develop complete descriptions of photophysical processes in OMIECs.

\section{Conclusions}

Ionic-electronic coupling is a fundamental process in OIMECs, and together with ion and electron transport determines the efficiency and functionality of a wide range of materials and devices. In this work we have probed the electrochemical doping mechanism of a redox active ProDOT-based polymer by monitoring linear-, transient-, and Raman spectroscopic variables in situ as a function of electrochemical potential. Our findings provide significant insight into the doping mechanism and evaluate the effects of the surrounding environment and intrinsic polymer structure on the doping process. A key finding of our work is the identification of a CT-state (at $1.58 \mathrm{eV}$ ) that precedes polaron formation and that shares a common ground state with the neutral transition but not with the electrochemically generated polarons. Using a combination of transient absorption and resonance Raman spectroscopy, we are able to show that the CT-state is stabilized by polarity, be it polarity induced by the electrolyte composition or the polymer structure. In addition, this CT-state is responsible for the quenching of the light-induced exciton population, via an ultrafast energy transfer mechanism.

Our resonance Raman results showed that polaron formation occurring in the early stages of the doping process also influence nearby neutral chains, where we observe an increase in both chain planarity and torsional order. In doped segments, polaron formation results in a modification of the chain conformation to a more planar structure with a relaxed intra-ring bonds, which seem 
to be beneficial for charge transport, as we observe a simultaneous increase in electrochemical conductance upon polaron formation. Our TA data confirmed that the electrochemically generated polarons do not share a common ground state with neutral state transition, contrary to what has been shown for an acid-doped donor-acceptor polymers. At high doping levels we observed bipolaron formation accompanied by a stiffening of both the inter- and intra-ring double bonds and a subsequent decrease in electrochemical conductance.

From the TA results, we also conclude that the excited state dynamics are sensitive to the surrounding environment, where substantially faster dynamics were observed in an aprotic organic electrolyte than in an aqueous electrolyte. By comparing polymers with the same backbone but different substituents in aqueous and organic electrolyte systems, we obtained evidence that subtle changes in molecular arrangement and chromophore coupling influences both the quenching and stabilization of photoexcitations. This highlights the importance and uniqueness of polymerelectrolyte interactions on the photophysical properties of OMIECs and showcases the dependence of these properties on the nature and composition of the surrounding electrolyte. Finally, with this work we identify an experimental toolset that is crucial for understanding in detail the nature of the energy states that could play a role in many technological applications, including (but not limited to) bioelectronics. These pointers can be effectively used to determine how chemical and morphological architectures can be engineered to enhance or suppress the states involved in the doping mechanism and mediating the working mechanism of electrochemical doping in conjugated polymers.

\section{Acknowledgement}

CS acknowledges funding from the National Science Foundation under grant number 1729737. LRS, AMÖ, and JRR gratefully acknowledge funding from the Air Force Office of Scientific Research (grant number FA9550-18-1-0184) and the Office of Naval Research (grant number N00014-20-1-2129). This material is based upon work supported by the National Science Foundation Graduate Research Fellowship under Grant No. DGE-1650044 (LRS). Resonance Raman spectroscopy was conducted at the Center for Nanophase Materials Sciences, which is a DOE Office of Science User Facility. The authors gratefully acknowledge Dr. Ilia Ivanov at the CNMS for his tremendous assistance with the Raman experiments. The authors also thank Dr. Ian 
Pelse for assistance with x-ray data acquisition and Dr. James Ponder, Jr. for his synthetic efforts. Use of the Stanford Synchrotron Radiation Lightsource, SLAC National Accelerator Laboratory, is supported by the U.S. Department of Energy, Office of Science, Office of Basic Energy Sciences under Contract No. DE-AC02-76SF00515. The authors thank Dr. Ajay Ram Srimath Kandada and Dr. Sophia Hayes for useful discussions.

\section{Acknowledgement}

The authors declare the following competing financial interest(s): Electrochromic polymer technology developed at the Georgia Institute of Technology has been licensed to NXN Licensing. JRR and AMÖ serve as a consultant to NXN Licensing. 


\section{References}

(1) Paulsen, B. D.; Tybrandt, K.; Stavrinidou, E.; Rivnay, J. Organic Mixed Ionic-Electronic Conductors. Nat. Mater. 2019, 19.

(2) Berggren, M.; Malliaras, G. G. How Conducting Polymer Electrodes Operate. Science 2019, 364 (6437), 233-234.

(3) Berggren, M.; Crispin, X.; Fabiano, S.; Jonsson, M. P.; Simon, D. T.; Stavrinidou, E.; Tybrandt, K.; Zozoulenko, I. V. Ion Electron-Coupled Functionality in Materials and Devices Based on Conjugated Polymers. Adv. Mater. 2019, 31 (22), 1805813.

(4) Snook, G. A.; Kao, P.; Best, A. S. Conducting-Polymer-Based Supercapacitor Devices and Electrodes. J. Power Sources 2011, 196 (1), 1-12.

(5) Wang, C.; Zheng, W.; Yue, Z.; Too, C. O.; Wallace, G. G. Buckled, Stretchable Polypyrrole Electrodes for Battery Applications. Adv. Mater. 2011, 23 (31), 3580-3584.

(6) Lang, A. W.; Ponder, J. F.; Österholm, A. M.; Kennard, N. J.; Bulloch, R. H.; Reynolds, J. R. Flexible, Aqueous-Electrolyte Supercapacitors Based on Water-Processable Dioxythiophene Polymer/Carbon Nanotube Textile Electrodes. J. Mater. Chem. A 2017, 5 (45), 23887-23897.

(7) Javier, A. E.; Patel, S. N.; Hallinan, D. T.; Srinivasan, V.; Balsara, N. P. Simultaneous Electronic and Ionic Conduction in a Block Copolymer: Application in Lithium Battery Electrodes. Angew. Chemie - Int. Ed. 2011, 50 (42), 9848-9851.

(8) Liang, Y.; Tao, Z.; Chen, J. Organic Electrode Materials for Rechargeable Lithium Batteries. Adv. Energy Mater. 2012, 2 (7), 742-769.

(9) Cirpan, A.; Argun, A. A.; Grenier, C. R. G.; Reeves, B. D.; Reynolds, J. R. Electrochromic Devices Based on Soluble and Processable Dioxythiophene Polymers . J. Mater. Chem. 2003, 13, 2418-2422.

(10) Andersson, P.; Forchheimer, R.; Tehrani, P.; Berggren, M. Printable All-Organic Electrochromic Active-Matrix Displays. Adv. Funct. Mater. 2007, 17 (16), 3074-3082.

(11) Mortimer, R. J.; Dyer, A. L.; Reynolds, J. R. Electrochromic Organic and Polymeric Materials for Display Applications. Displays 2006, 27 (1), 2-18.

(12) Van Reenen, S.; Janssen, R. A. J.; Kemerink, M. Dynamic Processes in Sandwich Polymer Light-Emitting Electrochemical Cells. Adv. Funct. Mater. 2012, 22 (21), 4547-4556.

(13) Li, Y.; Cao, Y.; Gao, J.; Wang, D.; Yu, G.; Heeger, A. J. Electrochemical Properties of Luminescent Polymers and Polymer Light-Emitting Electrochemical Cells. Synth. Met. 1999, 99 (3), 243-248.

(14) Simon, D. T.; Gabrielsson, E. O.; Tybrandt, K.; Berggren, M. Organic Bioelectronics: Bridging the Signaling Gap between Biology and Technology. Chem. Rev. 2016, 116 (21), 13009-13041. 
(15) Rivnay, J.; Inal, S.; Salleo, A.; Owens, R. M.; Berggren, M.; Malliaras, G. G. Organic Electrochemical Transistors. Nat. Rev. Mater. 2018, 3 (2), 17014-17086.

(16) Zeglio, E.; Inganäs, O. Active Materials for Organic Electrochemical Transistors. Adv. Mater. 2018, 30 (44), 1800941.

(17) Khodagholy, D.; Doublet, T.; Quilichini, P.; Gurfinkel, M.; Leleux, P.; Ghestem, A.; Ismailova, E.; Hervé, T.; Sanaur, S.; Bernard, C.; Malliaras, G. G. In Vivo Recordings of Brain Activity Using Organic Transistors. Nat. Commun. 2013, 4.

(18) Strakosas, X.; Bongo, M.; Owens, R. M. The Organic Electrochemical Transistor for Biological Applications. J. Appl. Polym. Sci. 2015, 132 (15), 41735.

(19) Leleux, P.; Rivnay, J.; Lonjaret, T.; Badier, J.-M.; Bénar, C.; Hervé, T.; Chauvel, P.; Malliaras, G. G. Organic Electrochemical Transistors for Clinical Applications. Adv. Healthc. Mater. 2014, 4 (1), 142-147.

(20) Isaksson, J.; Kjäll, P.; Nilsson, D.; Robinson, N.; Berggren, M.; Richter-Dahlfors, A. Electronic Control of $\mathrm{Ca} 2+$ Signalling in Neuronal Cells Using an Organic Electronic Ion Pump. Nat. Mater. 2007, 6 (9), 673-679.

(21) Williamson, A.; Rivnay, J.; Kergoat, L.; Jonsson, A.; Inal, S.; Uguz, I.; Ferro, M.; Ivanov, A.; Sjöström, T. A.; Simon, D. T.; Berggren, M.; Malliaras, G. G.; Bernard, C. Epilepsy Treatment: Controlling Epileptiform Activity with Organic Electronic Ion Pumps. Adv. Mater. 2015, 27 (20), 3097-3097.

(22) Picca, R. A.; Manoli, K.; Macchia, E.; Sarcina, L.; Di Franco, C.; Cioffi, N.; Blasi, D.; Österbacka, R.; Torricelli, F.; Scamarcio, G.; Torsi, L. Ultimately Sensitive Organic Bioelectronic Transistor Sensors by Materials and Device Structures’ Design. Adv. Funct. Mater. 2019, 30 (20), 1904513.

(23) Bernards, D. A.; Macaya, D. J.; Nikolou, M.; DeFranco, J. A.; Takamatsu, S.; Malliaras, G. G. Enzymatic Sensing with Organic Electrochemical Transistors. J. Mater. Chem. 2008, 18 (1), 116-120.

(24) Smela, E. Conjugated Polymer Actuators for Biomedical Applications. Adv. Mater. 2003, 15 (6), 481-494.

(25) Fannir, A.; Temmer, R.; Nguyen, G. T. M.; Cadiergues, L.; Laurent, E.; Madden, J. D. W.; Vidal, F.; Plesse, C. Linear Artificial Muscle Based on Ionic Electroactive Polymer: A Rational Design for Open-Air and Vacuum Actuation. Adv. Mater. Technol. 2019, 4 (2), 1800519.

(26) Giridharagopal, R.; Flagg, L. Q.; Harrison, J. S.; Ziffer, M. E.; Onorato, J.; Luscombe, C. K.; Ginger, D. S. Electrochemical Strain Microscopy Probes Morphology-Induced Variations in Ion Uptake and Performance in Organic Electrochemical Transistors. Nat. Mater. 2017, 16 (7), 737-742.

(27) Bischak, C. G.; Flagg, L. Q.; Yan, K.; Rehman, T.; Davies, D. W.; Quezada, R. J.; Onorato, J. W.; Luscombe, C. K.; Diao, Y.; Li, C.-Z.; Ginger, D. S. A Reversible Structural Phase Transition by Electrochemically-Driven Ion Injection into a Conjugated Polymer. J. Am. Chem. Soc. 2020, 142 (16), 7434-7442. 
(28) Thomas, E. M.; Brady, M. A.; Nakayama, H.; Popere, B. C.; Segalman, R. A.; Chabinyc, M. L. X-Ray Scattering Reveals Ion-Induced Microstructural Changes During Electrochemical Gating of Poly(3-Hexylthiophene). Adv. Funct. Mater. 2018, 28 (44), 1803687.

(29) Maddali, H.; House, K. L.; Emge, T. J.; O'Carroll, D. M. Identification of the Local Electrical Properties of Crystalline and Amorphous Domains in Electrochemically Doped Conjugated Polymer Thin Films. RSC Adv. 2020, 10 (36), 21454-21463.

(30) Rivnay, J.; Inal, S.; Collins, B. A.; Sessolo, M.; Stavrinidou, E.; Strakosas, X.; Tassone, C.; Delongchamp, D. M.; Malliaras, G. G. Structural Control of Mixed Ionic and Electronic Transport in Conducting Polymers. Nat. Commun. 2016, 7, 1-9.

(31) Savagian, L. R.; Österholm, A. M.; Ponder, J. J.; Barth, K. J.; Rivnay, J.; Reynolds, J. R. Balancing Charge Storage and Mobility in an Oligo(Ether) Functionalized Dioxythiophene Copolymer for Organic- and Aqueous- Based Electrochemical Devices and Transistors. Adv. Mater. 2018, 30 (50), 1804647.

(32) Roncali, J.; Shi, L. H.; Garreau, R.; Garnier, F.; Lemaire, M. Tuning of the Aqueous Electroactivity of Substituted Poly(Thiophene)s by Ether Groups. Synth. Met. 1990, 36, 267-273.

(33) Zhao, H.; Zhu, B.; Sekine, J.; Luo, S. C.; Yu, H. H. Oligoethylene-Glycol-Functionalized Polyoxythiophenes for Cell Engineering: Syntheses, Characterizations, and Cell Compatibilities. ACS Appl. Mater. Interfaces 2012, 4 (2), 680-686.

(34) Moser, M.; Ponder, J. J.; Wadsworth, A.; Giovannitti, A.; McCulloch, I. Materials in Organic Electrochemical Transistors for Bioelectronic Applications: Past, Present, and Future. Adv. Funct. Mater. 2019, 29 (21), 1807033.

(35) Giovannitti, A.; Sbircea, D.-T.; Inal, S.; Nielsen, C.; Bandiello, E.; Hanifi, D. A.; Sessolo, M.; Malliaras, G. G.; McCulloch, I.; Rivnay, J. Controlling the Mode of Operation of Organic Transistors through Side-Chain Engineering. Proc. Natl. Acad. Sci. 2016, 113 (43), 12017-12022.

(36) Flagg, L. Q.; Bischak, C. G.; Onorato, J. W.; Rashid, R. B.; Luscombe, C. K.; Ginger, D. S. Polymer Crystallinity Controls Water Uptake in Glycol Side-Chain Polymer Organic Electrochemical Transistors. J. Am. Chem. Soc. 2019, 141 (10), 4345-4354.

(37) Throughout the text, the term "polaron" is used to describe a charge carrier dressed by vibrational modes.

(38) Tsokkou, D.; Peterhans, L.; Cao, D. X.; Mai, C. K.; Bazan, G. C.; Nguyen, T. Q.; Banerji, N. Excited State Dynamics of a Self-Doped Conjugated Polyelectrolyte. Adv. Funct. Mater. 2019, 30 (9), 1906148.

(39) Prosa, T. J.; Winokur, M. J.; McCullough, R. D. Evidence of a Novel Side Chain Structure in Regioregular Poly(3-Alkylthiophenes). Macromolecules 1996, 29 (10), 3654-3656.

(40) Salleo, A. Impact of Organic Semiconductor Microstructure on Transport: Basic Concepts. In The WSPC Reference on Organic Electronics: Organic Semiconductors; Bredas, J.-L., Marder, S. R., Eds.; WORLD SCIENTIFIC, 2016; pp 293-323. 
(41) Lim, E.; Peterson, K. A.; Su, G. M.; Chabinyc, M. L. Thermoelectric Properties of Poly(3Hexylthiophene) (P3HT) Doped with 2,3,5,6-Tetrafluoro-7,7,8,8Tetracyanoquinodimethane (F4TCNQ) by Vapor-Phase Infiltration. Chem. Mater. 2018, 30 (3), 998-1010.

(42) Duong, D. T.; Wang, C.; Antono, E.; Toney, M. F.; Salleo, A. The Chemical and Structural Origin of Efficient P-Type Doping in P3HT. Org. Electron. 2013, 14 (5), 1330-1336.

(43) Kayunkid, N.; Uttiya, S.; Brinkmann, M. Structural Model of Regioregular Poly(3Hexylthiophene) Obtained by Electron Diffraction Analysis. Macromolecules 2010, 43 (11), 4961-4967.

(44) Noriega, R.; Rivnay, J.; Vandewal, K.; Koch, F. P. V.; Stingelin, N.; Smith, P.; Salleo, A. A General Relationship between Disorder, Aggregation and Charge Transport in Conjugated Polymers. Nat. Mater. 2013, 12, 1038-1044.

(45) Duong, D. T.; Toney, M. F.; Salleo, A. Role of Confinement and Aggregation in Charge Transport in Semicrystalline Polythiophene Thin Films. Phys. Rev. B - Condens. Matter Mater. Phys. 2012, 86 (20), 1-5.

(46) Skompska, M.; Szkurlat, A. The Influence of the Structural Defects and Microscopic Aggregation of Poly(3-Alkylthiophenes) on Electrochemical and Optical Properties of the Polymer Films: Discussion of an Origin of Redox Peaks in the Cyclic Voltammograms. Electrochim. Acta 2001, 46 (26-27), 4007-4015.

(47) Sweetnam, S.; Graham, K. R.; Ngongang Ndjawa, G. O.; Heumüller, T.; Bartelt, J. A.; Burke, T. M.; Li, W.; You, W.; Amassian, A.; McGehee, M. D. Characterization of the Polymer Energy Landscape in Polymer:Fullerene Bulk Heterojunctions with Pure and Mixed Phases. J. Am. Chem. Soc. 2014, 136 (Cv), 14078-14088.

(48) Österholm, A. M.; Ponder, J. F.; De Keersmaecker, M.; Shen, D. E.; Reynolds, J. R. Disentangling Redox Properties and Capacitance in Solution-Processed Conjugated Polymers. Chem. Mater. 2019, 31 (8), 2971-2982.

(49) Harris, J. K.; Neelamraju, B.; Ratcliff, E. L. Intersystem Subpopulation Charge Transfer and Conformational Relaxation Preceding in Situ Conductivity in Electrochemically Doped Poly(3-Hexylthiophene) Electrodes. Chem. Mater. 2019, 31 (17), 6870-6879.

(50) Wang, C.; Duong, D. T.; Vandewal, K.; Rivnay, J.; Salleo, A. Optical Measurement of Doping Efficiency in Poly(3-Hexylthiophene) Solutions and Thin Films. Phys. Rev. B 2015, $91(8), 85205-85207$.

(51) Spano, F. C.; Silva, C. H- and J-Aggregate Behavior in Polymeric Semiconductors. Annu. Rev. Phys. Chem. 2014, 65 (1), 477-500.

(52) Clark, J.; Chang, J.-F.; Spano, F. C.; Friend, R. H.; Silva, C. Determining Exciton Bandwidth and Film Microstructure in Polythiophene Films Using Linear Absorption Spectroscopy. Appl. Phys. Lett. 2009, 94 (16), 163304-163306.

(53) Brédas, J. L.; Thémans, B.; Fripiat, J. G.; André, J. M.; Chance, R. R. Highly Conducting Polyparaphenylene, Polypyrrole, and Polythiophene Chains: An Ab Initio Study of the Geometry and Electronic-Structure Modifications upon Doping. Phys. Rev. B 1984, 29 (12), 
6761-6773.

(54) Brédas, J. L.; Street, G. B. Polarons, Bipolarons, and Solitons in Conducting Polymers. Acc. Chem. Res. 1985, 18 (10), 309-315.

(55) Mariani, M. M.; Deckert, V. Raman Spectroscopy: Principles, Benefits, and Applications. In Methods in Physical Chemistry; Wiley-VCH Verlag GmbH \& Co. KGaA: Weinheim, Germany, 2012; pp 419-444.

(56) In this work, we were unable to perform Raman resonant with the sub-gap state at $1.11 \mathrm{eV}$. Therefore, we restrict our analyses to the unique vibrational characteristics couplied to the neutral exciton and the sub-gap state at $1.58 \mathrm{eV}$.

(57) Chiu, W. W.; Travaš-Sejdić, J.; Cooney, R. P.; Bowmaker, G. A. Studies of Dopant Effects in Poly(3,4-Ethylenedioxythiophene) Using Raman Spectroscopy. J. Raman Spectrosc. 2006, 37 (12), 1354-1361.

(58) Garreau, S.; Louarn, G.; Buisson, J. P.; Froyer, G.; Lefrant, S. In Situ Spectroelectrochemical Raman Studies of Poly(3,4-Ethylenedioxythiophene) (PEDT). Macromolecules 1999, 32 (20), 6807-6812.

(59) Gao, Y.; Grey, J. K. Resonance Chemical Imaging of Polythiophene/Fullerene Photovoltaic Thin Films: Mapping Morphology-Dependent Aggregated and Unaggregated $\mathrm{C}=\mathrm{C}$ Species. J. Am. Chem. Soc. 2009, 131 (28), 9654-9662.

(60) Tsoi, W. C.; James, D. T.; Kim, J. S.; Nicholson, P. G.; Murphy, C. E.; Bradley, D. D. C.; Nelson, J.; Kim, J. S. The Nature of In-Plane Skeleton Raman Modes of P3HT and Their Correlation to the Degree of Molecular Order in P3HT:PCBM Blend Thin Films. J. Am. Chem. Soc. 2011, 133 (25), 9834-9843.

(61) Dyson, M. J.; Lariou, E.; Martin, J.; Li, R.; Erothu, H.; Wantz, G.; Topham, P. D.; Dautel, O. J.; Hayes, S. C.; Stavrinou, P. N.; Stingelin, N. Managing Local Order in Conjugated Polymer Blends via Polarity Contrast. Chem. Mater. 2019, acs.chemmater.8b05259.

(62) Cabanillas-Gonzalez, J.; Grancini, G.; Lanzani, G. Pump-Probe Spectroscopy in Organic Semiconductors: Monitoring Fundamental Processes of Relevance in Optoelectronics. Adv. Mater. 2011, 23 (46), 5468-5485.

(63) Bargigia, I.; Zucchetti, E.; Kandada, A. R. S.; Moreira, M.; Bossio, C.; Wong, W. P. D.; Miranda, P. B.; Decuzzi, P.; Soci, C.; D’Andrea, C.; Lanzani, G. The Photophysics of Polythiophene Nanoparticles for Biological Applications. ChemBioChem 2019, 20 (4), 532-536.

(64) Hodgkiss, J. M.; Albert-Seifried, S.; Rao, A.; Barker, A. J.; Campbell, A. R.; Marsh, R. A.; Friend, R. H. Exciton-Charge Annihilation in Organic Semiconductor Films. Adv. Funct. Mater. 2012, 22 (8), 1567-1577.

(65) Papke, B. L.; Ratner, M. A.; Shriver, D. F. Vibrational Spectroscopy and Structure of Polymer Electrolytes, Poly(Ethylene Oxide) Complexes of Alkali Metal Salts. J. Phys. Chem. Solids 1981, 42 (6), 493-500.

(66) Marsella, M. J.; Swager, T. M. Designing Conducting Polymer-Based Sensors: Selective 
Ionochromic Response in Crown Ether Containing Polythiophenes. J. Am. Chem. Soc. 1993, 115 (25), 12214-12215.

(67) Wright, P. V. Electrical Conductivity in Ionic Complexes of Poly(Ethylene Oxide). Br. Polym. J. 1975, 7 (5), 319-327.

(68) Moser, M.; Thorley, K. J.; Moruzzi, F.; Ponder, J. J.; Maria, I. P.; Giovannitti, A.; Inal, S.; McCulloch, I. Highly Selective Chromoionophores for Ratiometric $\mathrm{Na}+$ Sensing Based on an Oligoethyleneglycol Bridged Bithiophene Detection Unit. J. Mater. Chem. C 2019, 7 (18), 5359-5365. 